\title{
A NEW LOOK AT MOURRE'S COMMUTATOR THEORY
}

\author{
SYLVAIN GOLÉNIA AND THIERRY JECKO
}

\begin{abstract}
Mourre's commutator theory is a powerful tool to study the continuous spectrum of self-adjoint operators and to develop scattering theory. We propose a new approach of its main result, namely the derivation of the limiting absorption principle (LAP) from a so called Mourre estimate. We provide a new interpretation of this result.
\end{abstract}

\section{Contents}

1. Introduction 2

2. A new approach of the LAP. 5

2.1. Basic facts and notation 5

2.2. Local regularity and main result 6

2.3. Special sequences and the LAP 8

2.4. A Virial-like Theorem. 10

2.5. Sketch of our proof and interpretation. 10

3. A new proof of the LAP. 11

3.1. Proof of Theorem $2.7 \quad 11$

3.2. A "large $|A|$ " estimate. 12

3.3. Absence of mass. 15

4. The LAP for the reduced resolvent. 16

\begin{tabular}{lll}
\hline 4.1. & Motivation & 16
\end{tabular}

Date: July 28, 2018.

2000 Mathematics Subject Classification. 47A40,47B25, 81U99.

Key words and phrases. Mourre's commutator theory, Mourre estimate, limiting absorption principle, continuous spectrum. 
$\begin{array}{lll}\text { 4.2. Eigenvectors' regularity. } & 17\end{array}$

4.3. Proof of Theorem $1.4 \quad 17$

4.4. Proofs of Theorem $4.3 \quad 18$

4.5. An artificial but instructive example. 20

\begin{tabular}{|lll}
\hline Appendix A. Svmbolic calculus. & 21
\end{tabular}

Appendix B. Commutator expansions. 22

\begin{tabular}{|lll}
\hline Appendix C. Technical estimates. & 24
\end{tabular}

References 26

\section{INTRODUCTION}

In the beginning of the eigthies, Mourre's commutator theory was developed in [M] to show absolute continuity of the continuous spectrum of $N$-body Schrödinger operators and to study their scattering theory (cf. $\mathrm{ABG}, \mathrm{HuS}]$ ). In particular, one wanted to show their asymptotic completeness and the Mourre estimate (cf. (1.1)) played a crucial role in the proof (cf. DG, $\mathrm{HuS}$ ). Now, Mourre's commutator theory is fundamental tool to develop the stationary scattering theory of general self-adjoint operators. We refer to $\mathrm{ABG}, \mathrm{DG}$ for details. We point out that the theory is still used (see [BCHM, CGH, DJ, GGo, for instance) and that there were new developements to apply it to quantum field theory (cf. GGM1, GGM2]). The theory uses a so called differential inequality technics, that is quite magic and mysterious (to us at least). In this paper, we propose a new approach and interpretation of the theory. Since the original method has been developed to a rather sophisticated level (cf. ABG, GGM1, S] ), we did not try to optimize our approach and to give new results, but to focus on an intermediate, interesting situation. However, Theorem 1.4 gives an extension of results in [C, CGH]. We point out that our new approach of Mourre's commutator theory is an adaptation of a strategy to get semiclassical resolvent estimates for Schrödinger operators. This strategy was introduced by the second author in [J1] and further used in [CJ, J2].

To enter into the details of our approach, we need some notation and basic notions (see Subsection 2.1 for details). We consider two selfadjoint (unbounded) operators $H$ and $A$ acting in some complex Hilbert space $\mathscr{H}$. Let $\|\cdot\|$ denote the norm of bounded operators on $\mathscr{H}$. We 
shall study spectral properties of $H$ with the help of $A$. Since the commutator $[H, i A]$ is going to play a central role in the theory, we need some regularity of $H$ with respect to $A$ to give an appropriate sense to this commutator. Since $H$ is self-adjoint, its spectrum is included in $\mathbb{R}$. Given $k \in \mathbb{N}$, we say that $H \in \mathcal{C}^{k}(A)$ if for some (and thus for all) $z \notin \mathbb{R}$, for all $f \in \mathscr{H}$, the map $\mathbb{R} \ni t \mapsto e^{i t A}(H-z)^{-1} e^{-i t A} f \in \mathscr{H}$ has the usual $\mathcal{C}^{k}$ regularity. Let $H \in \mathcal{C}^{1}(A)$ and $\mathcal{I}$ be a bounded interval of $\mathbb{R}$. We say that the Mourre estimate holds true for $H$ on $\mathcal{I}$ if there exist $c>0$ and a compact operator $K$ such that

$$
E_{\mathcal{I}}(H)[H, i A] E_{\mathcal{I}}(H) \geq c E_{\mathcal{I}}(H)+K,
$$

in the form sense on $\mathscr{H} \times \mathscr{H}$. Here $E_{\mathcal{I}}(H)$ denotes the spectral measure of $H$ above $\mathcal{I}$.

Remark 1.1. Let $f \in \mathscr{H}$ and $\lambda \in \mathcal{I}$ with $H f=\lambda f$. Then $E_{\mathcal{I}}(H) f=f$. Assume that $H \in \mathcal{C}^{1}(A)$. The Virial theorem (cf. ABG, Proposition 7.2.10]) implies that $\langle f,[H, i A] f\rangle=0$. If (1.1) holds true with $K=0$ then $f$ must be zero and there is no eigenvalue in $\mathcal{I}$. If (1.1) holds true then the total multiplicity of the eigenvalues in $\mathcal{I}$ is finite (cf. $\mathrm{ABG}$, Corollary 7.2.11]). A weaker version of this result is due to Mourre in [M]. For a general discussion on the Virial theorem see GGé.

The main aim of Mourre's commutator theory is to show the limiting absorption principle (LAP) on some bounded interval $\mathcal{I}$ in $\mathbb{R}$. Given such a $\mathcal{I}$ and $s \geq 0$, we say that the LAP, respectively to the triplet $(\mathcal{I}, s, A)$, holds true for $H$ if

$$
\sup _{\operatorname{Re} z \in \mathcal{I}, \operatorname{Im} z \neq 0}\left\|\langle A\rangle^{-s}(H-z)^{-1}\langle A\rangle^{-s}\right\|<\infty .
$$

Theorem 1.2. Let $H \in \mathcal{C}^{2}(A)$, $\mathcal{I}$ be a bounded, open interval, and $s>1 / 2$. Assume the strict Mourre estimate, $i$. e. (1.1) with $K=0$, holds true. Then, for any closed subinterval $\mathcal{I}^{\prime}$ of $\mathcal{I}$, the $L A P$ for $H$ respectively to $\left(\mathcal{I}^{\prime}, s, A\right)$ holds true.

Remark 1.3. Assume the Mourre estimate (1.1) holds true on $\mathcal{I}$ with $K \neq 0$. Then, on small enough intervals outside the point spectrum $\sigma_{p p}(H)$ of $H$, which is finite by Remark 1.1. the strict Mourre estimate (1.1) with $K=0$ holds true (cf. ABG) and Theorem 1.2 applies there. Putting all together, this yields the LAP on any compact subset of $\mathcal{I} \backslash \sigma_{p p}(H)$.

Compared with previous results, we do not need that the domain $\mathcal{D}(H)$ of $H$ is invariant under the $C_{0}$-group generated by $A$ (i.e. the propagator of $A$ ) or that $H$ has a spectral gap (cf. ABG]). The main reason 
for this comes from the fact that we do not work with $H$ itself but with a local version of $H$, which is a bounded operator. This explains also why we can replace the global regularity assumption $H \in \mathcal{C}^{2}(A)$ by a local one and get a stronger result, namely Theorem 2.7. The latter is covered by Sahbani's result in [S] (cf. Remark 2.8). Motivations for Theorem 2.7] are given in Remarks 2.8 and 2.9. In Subsection 2.5, we give a sketch of the proof of Theorem 2.7 and present our interpretation of Mourre's commutator theory, which is close to the interpretation of Remark 1.1. We do not use the usual differential inequality technics.

In some sense, Theorem 1.2 (and also Theorem 2.7) is not satisfactory (cf. Subsection 4.1) and one wishes to replace the resolvent $(H-z)^{-1}$ in (1.2) by the reduced resolvent, namely $(H-z)^{-1} P^{\perp}$, where $P^{\perp}=$ $1-P$, and $P$ is the orthogonal projection onto the pure point spectral subspace of $H$. For $s \geq 0$, we say that the reduced LAP, respectively to the triplet $(\mathcal{I}, s, A)$, holds true for $H$ if

$$
\sup _{\operatorname{Re} z \in \mathcal{I}, \operatorname{Im} z \neq 0}\left\|\langle A\rangle^{-s}(H-z)^{-1} P^{\perp}\langle A\rangle^{-s}\right\|<\infty .
$$

Theorem 1.4. Let $H \in \mathcal{C}^{2}(A), \mathcal{I}$ be a bounded, open interval and let $s>1 / 2$. Assume the Mourre estimate (1.1) holds true on $\mathcal{I}$. Assume also that the range $\operatorname{Ran} P E_{\mathcal{I}}$ of $P E_{\mathcal{I}}$ is included in the domain $\mathcal{D}\left(A^{2}\right)$ of $A^{2}$. Then, for all closed interval $\mathcal{I}^{\prime}$ included in the interior of $\mathcal{I}$, the reduced $L A P(1.3)$, respectively to $\left(\mathcal{I}^{\prime}, s, A\right)$, holds true for $H$.

A similar result appears in [CGH]. The authors assume a stronger regularity (essentially like $H \in \mathcal{C}^{4}(A)$ ) that implies $\operatorname{Ran} P E_{\mathcal{I}} \subset \mathcal{D}\left(A^{2}\right)$, by $[\mathrm{C}$, and then show (1.3). The latter result and Theorem 1.4 actually work with weaker, "local" assumptions as shown in Proposition 4.1 and Theorem 4.3. As mentioned before, this local version of the result might be important (cf. Remarks 2.8 and 2.9). In [CGH], some Hölder continuity of the boundary values of the reduced resolvent $\lim _{\epsilon \rightarrow 0^{+}}\langle A\rangle^{-s}(H-\lambda-i \epsilon)^{-1} P^{\perp}\langle A\rangle^{-s}$ is obtained. In Remark 4.6] we explain how to get this under "local" assumptions, using [S].

We point out that our proofs of Theorems 1.4 and 4.3 is a quite immediate generalization of our proofs of Theorems 1.2 and 2.7. We also give an alternative proof of Theorem 4.3 which is close to the corresponding proof in [CGH]. Notice further that, Theorems 4.3 works under a projected Mourre estimate (4.28), that is weaker than (1.1). In Subsection 4.5, we illustrate this difference with an artificial but interesting example, for which the reduced LAP (1.3) holds true and the usual Mourre estimate (1.1) is false. This example is however related to the situation in [DJ]. 
Paper's organisation: In Section 2, we introduce the main tools of our new approach. Admitting Theorem 2.7, we prove Theorem 1.2 in Subsection 2.2. Section [3] is devoted to the proof of Theorem 2.7. In Section 4, we prove Theorems 1.4 and 4.3 on the reduced resolvent. Technical tools are collected in Appendices A, B, and C,

Acknowledgments: We would like to thank Jan Dereziński, Vladimir Georgescu, Jacob S. Møller, and Francis Nier for helpful discussions. The two authors were partially supported by the contract MERG-CT2004-006375 funded by the European commission and the first one was also supported by the Postdoctoral Training Program HPRN-CT2002-0277. The second author thanks the members of the Institut of Mathematics "Simion Stoilow" of the Romanian Academy in Bucharest for their kind hospitality.

\section{A NEW APPROACH OF THE LAP.}

We explain in this section our strategy to prove Theorem 2.7 below, a stronger version of Theorem 1.2.

2.1. Basic facts and notation. In this subsection, we introduce some notation and recall known basic results. We refer to $\mathrm{ABG}$ for details.

In the text, we use the letter $\mathcal{I}$ to denote an interval of $\mathbb{R}$. For such a $\mathcal{I}$, we denote by $\overline{\mathcal{I}}$ (resp. $\stackrel{\mathcal{I}}{\text { ) }}$ its closure (resp. its interior). The scalar product $\langle\cdot, \cdot\rangle$ in $\mathscr{H}$ is right linear and $\|\cdot\|$ denotes the corresponding norm and also the norm of bounded operators on $\mathscr{H}$. If $T$ is a bounded operator on $\mathscr{H}$ and $k \in \mathbb{N}$, we say that $T \in \mathcal{C}^{k}(A)$ if, for all $f \in \mathscr{H}$, the map $\mathbb{R} \ni t \mapsto e^{i t A} T e^{-i t A} f \in \mathscr{H}$ has the usual $\mathcal{C}^{k}$ regularity. It turns out that $T \in \mathcal{C}^{k}(A)$ if and only if, for a $z$ outside the spectrum of $T$, $(T-z)^{-1} \in \mathcal{C}^{k}(A)$. For such $T, T \in \mathcal{C}^{1}(A)$ if and only if the form $[T, A]$ defined on $\mathcal{D}(A) \times \mathcal{D}(A)$ extends to a bounded operator $\operatorname{ad}_{A}^{1}(T)=[T, A]$ if and only if $T$ preserves $\mathcal{D}(A)$ and the operator $T A-A T$, defined on $\mathcal{D}(A)$, extends to a bounded operator in $\mathscr{H}$. Furthermore $T \in \mathcal{C}^{k}(A)$ if and only if the iterated commutator $\operatorname{ad}_{A}^{p}(T):=\left[\operatorname{ad}_{A}^{p-1}(T), A\right]$ are bounded for $p \leq k$. In particular, for $T \in \mathcal{C}^{1}(A), T \in \mathcal{C}^{2}(A)$ if and only if $[T, A] \in \mathcal{C}^{1}(A)$. For unbounded self-adjoint operator, we defined the $\mathcal{C}^{k}(A)$ regularity in Section 11. Let $H$ is (unbounded) self-adjoint operator and $\mathcal{I}$ a bounded interval. Recall that $E_{\mathcal{I}}(H)$ denotes the spectral projection of $H$ above $\mathcal{I}$. If $H \in \mathcal{C}^{1}(A)$ then the form $[H, i A]$ defined on $(\mathcal{D}(H) \cap \mathcal{D}(A)) \times(\mathcal{D}(H) \cap \mathcal{D}(A))$ extend to a bounded operator from $\mathcal{D}(H)$ to its dual for the graph norm. In particular, (1.1) 
makes sense. A justification of Remark 1.3 can be found in ABG but we give it in the proof of Theorem 1.4 (for $P=0$ ). The following propositions and remark will be useful later.

Proposition 2.1. For $f, g \in \mathcal{D}(A)$, the finite rank operator $|f\rangle\langle g|$ : $h \rightarrow\langle g, h\rangle \cdot f$ belongs to $\mathcal{C}^{1}(A)$ and $[|f\rangle\langle g|, A]=|f\rangle\langle A g|-| A f\rangle\langle g|$. In particular, if $f, g \in \mathcal{D}\left(A^{2}\right),|f\rangle\langle g| \in \mathcal{C}^{2}(A)$. If $P$ is a finite rank projection, the range of which is included in $\mathcal{D}\left(A^{k}\right)$ with $k \in \mathbb{N}$, then $P \in \mathcal{C}^{k}(A)$

Proof. Since $R:=|f\rangle\langle g|$ preserves $\mathcal{D}(A)$, the commutator $[|f\rangle\langle g|, A]$ is well defined on $\mathcal{D}(A)$ and $[|f\rangle\langle g|, A]=|f\rangle\langle A g|-| A f\rangle\langle g|$, which extends to a bounded operator. Thus $R \in \mathcal{C}^{1}(A)$. Applying the first result to $[R, A],|f\rangle\langle g| \in \mathcal{C}^{2}(A)$ if $f, g \in \mathcal{D}\left(A^{2}\right)$. Since $P=\sum_{1 \leq n \leq N}\left|f_{n}\right\rangle\left\langle f_{n}\right|$ with $N \in \mathbb{N}$, an induction argument gives the last result.

Proposition 2.2. Let $\left(T_{n}\right)_{n}$ be a sequence of bounded operators such that, $T_{n} \in \mathcal{C}^{1}(A)$, for all $n$, and such that there exist bounded $S, T$ such that $T_{n} \rightarrow T$ and $\left[T_{n}, A\right] \rightarrow S$ in the norm topology. Then $T \in \mathcal{C}^{1}(A)$ and $S=[T, A]$.

Proof. See Lemma 2.5 in GGM1.

Remark 2.3. The LAP, respectively to $(\mathcal{I}, 0, A)$, holds true for $H$ if and only if $H$ has no spectrum in $\mathcal{I}$. The LAP for $H$, respectively to $(\mathcal{I}, s, A)$, implies the LAP for $H$, respectively to $\left(\mathcal{I}, s^{\prime}, A\right)$, for any $s^{\prime} \geq$ $s$. For $H=-\Delta$ the Laplace operator in $\mathbb{R}^{d}$ and $A$ the multiplication operator by $\langle x\rangle$, it is known that LAP for $H$, respectively to $(\mathcal{I}, s, A)$, holds true if and only if $s>1 / 2$ (cf. [H] $)$.

2.2. Local regularity and main result. In Theorem 1.2 the LAP (1.2) and the Mourre estimate (1.1) are localized in $H$. It is quite natural to try to replace $H$ and the global assumption $H \in \mathcal{C}^{2}(A)$ by some local version. By $\mathrm{ABG}$, we have

Proposition 2.4. Let $\varphi \in \mathcal{C}_{c}^{\infty}(\mathbb{R})$. Suppose $H \in \mathcal{C}^{k}(A)$ for a certain $k \in \mathbb{N}$. Then, $\varphi(H) \in \mathcal{C}^{k}(A)$.

For any $\tau \in \mathcal{C}_{c}^{\infty}(\mathbb{R})$, we define the bounded operator

$$
H_{\tau}:=H \tau(H) \text {. }
$$

It turns out that we can deduce the LAP for $H$ respectively to $(\mathcal{I}, s, A)$ from the LAP for $H_{\tau}$ respectively to $(\mathcal{I}, s, A)$, if $\tau=1$ near $\mathcal{I}$, as seen in Proposition 2.13 below. Thus $H_{\tau}$ is a good local (and bounded) version of $H$. From $\underline{S}$, Proposition 2.1], we pick the following 
Lemma 2.5. Let $\mathcal{I}$ be bounded, open interval. Suppose that $H \in \mathcal{C}^{1}(A)$ and that the Mourre estimate (1.1) holds true on $\mathcal{I}$. Take $\theta \in \mathcal{C}_{c}^{\infty}(\mathcal{I})$ and $\tau \in \mathcal{C}_{c}^{\infty}(\mathbb{R})$ such that $\tau \theta=\theta$. Then $H_{\tau} \in \mathcal{C}^{1}(A)$ and

$$
\theta(H)\left[H_{\tau}, i A\right] \theta(H) \geq c \theta^{2}(H)+\theta(H) K \theta(H) .
$$

Proof. By Proposition 2.4, $H_{\tau} \in \mathcal{C}^{1}(A)$. For $f \in \mathcal{D}(A \theta(H))$,

$$
\langle H \theta(H) f, i A \theta(H) f\rangle-\langle i A \theta(H) f, H \theta(H) f\rangle \geq c\|\theta(H) f\|^{2}+\langle f, K f\rangle .
$$

Now, use that $H \theta(H)=H \tau(H) \theta(H)$. Finally, $\mathcal{D}(A \theta(H))$ is dense in $\mathscr{H}$ since $\theta(H) A$ is closed with a dense domain.

Remark 2.6. In general, one should not expect a "real" Mourre estimate for $H_{\tau}$ of the form

$$
\varphi\left(H_{\tau}\right)\left[H_{\tau}, i A\right] \varphi\left(H_{\tau}\right) \geq c \varphi^{2}\left(H_{\tau}\right)+K
$$

for a certain function $\varphi$ which satifies the same hypothesis as $\theta$ in Lemma 2.5. Indeed, since $0 \in \operatorname{supp} \theta$, there is no such function $\varphi$ such that $\varphi(t \tau(t))=\theta(t)$ for all $t \in \mathbb{R}$.

Given an open interval $\mathcal{I}$ and $k \in \mathbb{N}$, we say that $H$ is locally of class $\mathcal{C}^{k}(A)$ on $\mathcal{I}$, we write $H \in \mathcal{C}_{\mathcal{I}}^{k}(A)$, if, for all $\varphi \in \mathcal{C}_{c}^{\infty}(\mathcal{I}), \varphi(H) \in \mathcal{C}^{k}(A)$. This is a local version of the regularity $\mathcal{C}^{k}(A)$ which was already used in $[$ ].

Proof of Theorem 1.2; Let $\mathcal{I}^{\prime \prime}$ be open such that $\overline{\mathcal{I}} \subset \mathcal{I}^{\prime \prime}$. By Lemma 2.4. $H \in \mathcal{C}_{\mathcal{I}^{\prime \prime}}^{2}(A)$. Let $\tau \in \mathcal{C}_{c}^{\infty}\left(\mathcal{I}^{\prime \prime}\right)$ such that $\tau=1$ near $\mathcal{I}$. Let $\mathcal{I}_{1}$ be closed such that $\mathcal{I}^{\prime} \subset \stackrel{\circ}{\mathcal{I}}_{1}$ and $\mathcal{I}_{1} \subset \mathcal{I}$. Let $\theta \in \mathcal{C}_{c}^{\infty}(\mathcal{I})$ such that $\theta=1$ on $\mathcal{I}_{1}$. By Lemma 2.5 and (1.1), we derive (2.5), which implies

$$
E_{\mathcal{I}_{1}}(H)\left[H_{\tau}, i A\right] E_{\mathcal{I}_{1}}(H) \geq c E_{\mathcal{I}_{1}}(H)+0,
$$

since $\theta=1$ on $\mathcal{I}_{1}$. Thus Theorem 2.7 below applies yielding the LAP for $H$ respectively to $\left(\mathcal{I}^{\prime}, s, A\right)$.

So the proof of Theorem 1.2 reduces to the proof of the following stronger result, which is our main result.

Theorem 2.7. Let $\mathcal{I}$ be a bounded, open interval. Let $\mathcal{I}^{\prime \prime}$ be an open interval such that $\overline{\mathcal{I}} \subset \mathcal{I}^{\prime \prime}$. Let $H \in \mathcal{C}_{\mathcal{I}^{\prime \prime}}^{2}(A)$ and $\tau \in \mathcal{C}_{c}^{\infty}\left(\mathcal{I}^{\prime \prime}\right)$ such that $\tau=1$ near $\mathcal{I}$. Suppose the strict Mourre estimate

$$
E_{\mathcal{I}}(H)\left[H_{\tau}, i A\right] E_{\mathcal{I}}(H) \geq c E_{\mathcal{I}}(H) \text {, with } c>0,
$$

holds true. Then, for any $s>1 / 2$ and any compact interval $\mathcal{I}^{\prime}$ with $\mathcal{I}^{\prime} \subset \stackrel{\circ}{\mathcal{I}}$, the LAP respectively to $\left(\mathcal{I}^{\prime}, s, A\right)$ holds true for $H_{\tau}$ and $H$. 
Proof. See Subsection 3.1] (and Subsection 2.5 for a sketch).

Remark 2.8. In $[\mathrm{S}$, the previous result is proved under a weaker local regularity assumption (slightly stronger than $\mathcal{C}_{\mathcal{I}^{\prime \prime}}^{1}(A)$ ), using Mourre's differential inequality technics. Furthermore, an example of multiplication operator $H$ and of conjugate operator $A$ is given such that $H \notin \mathcal{C}^{1}(A)$ but $H \in \mathcal{C}_{\mathcal{I}}^{1}(A)$, for some $\mathcal{I}$.

Remark 2.9. Assume that Theorem 1.2 applies to some operators $H$ and $A$ on some interval $\mathcal{I}$. Let $\mathcal{I}^{\prime \prime}$ be open such that $\overline{\mathcal{I}} \subset \mathcal{I}^{\prime \prime}$. Let $\varphi: \mathbb{R} \longrightarrow \mathbb{R}$ be a borelian, increasing function such that, for all $t \in \mathcal{I}^{\prime \prime}$, $\varphi(t)=t$. Then Theorem 2.7 applies with $H$ replaced by $\varphi(H)$. Since $\varphi$ may be irregular outside $\mathcal{I}^{\prime \prime}$, we do not know if $\varphi(H) \in \mathcal{C}^{1}(A)$, so if Theorem 1.2 applies to $\varphi(H)$.

2.3. Special sequences and the LAP. In this subsection, we introduce our main tool and its properties. We proceed like in J1] and use the terminology appearing in this semi-classical setting.

Definition 2.10. A special sequence $\left(f_{n}, z_{n}\right)_{n}$ for $H$ associated to $(\mathcal{I}, s, A)$, as in (1.2) , is a sequence $\left(f_{n}, z_{n}\right)_{n} \in(\mathcal{D}(H) \times \mathbb{C})^{\mathbb{N}}$ such that, for certain $\lambda \in \mathcal{I}$ and $\eta \geq 0, \mathcal{I} \ni \operatorname{Re}\left(z_{n}\right) \rightarrow \lambda, 0 \neq \operatorname{Im}\left(z_{n}\right) \rightarrow 0$, $\left\|\langle A\rangle^{-s} f_{n}\right\| \rightarrow \eta,\left(H-z_{n}\right) f_{n} \in \mathcal{D}\left(\langle A\rangle^{s}\right)$, and $\left\|\langle A\rangle^{s}\left(H-z_{n}\right) f_{n}\right\| \rightarrow 0$. The limit $\eta$ is called the mass of the special sequence.

We give the link between this notion and the LAP in

Proposition 2.11. Given $s \geq 0$ and a compact interval $\mathcal{I}$, the $L A P$ for $H$ respectively to $(\mathcal{I}, s, A)$ is false if and only if there exists a special sequence $\left(f_{n}, z_{n}\right)_{n}$ for $H$ associated to $(\mathcal{I}, s, A)$ with a positive mass.

Proof. Suppose the LAP to be false. There exist a sequence $\left(k_{n}\right)_{n}$ of nonnegative numbers, going to infinity, a sequence $\left(g_{n}\right)_{n}$ of non-zero elements of $\mathscr{H}$, and a sequence $\left(z_{n}\right)_{n}$ of complex numbers such that $\operatorname{Re}\left(z_{n}\right) \in \mathcal{I}, 0 \neq \operatorname{Im}\left(z_{n}\right) \rightarrow 0$, and

$$
\left\|\langle A\rangle^{-s}\left(H-z_{n}\right)^{-1}\langle A\rangle^{-s} g_{n}\right\|=k_{n}\left\|g_{n}\right\|=1 \text {. }
$$

Setting $f_{n}=\left(H-z_{n}\right)^{-1}\langle A\rangle^{-s} g_{n}, f_{n} \in \mathcal{D}(H),\left(H-z_{n}\right) f_{n} \in \mathcal{D}\left(\langle A\rangle^{s}\right)$, and, by (2.7),

$$
\left\|\langle A\rangle^{-s} f_{n}\right\|=1 \text { and }\left\|\langle A\rangle^{s}\left(H-z_{n}\right) f_{n}\right\|=1 / k_{n} \rightarrow 0 .
$$

Up to a subsequence, we can assume that $\operatorname{Re}\left(z_{n}\right) \rightarrow \lambda \in \mathcal{I}$. Now, we assume the LAP true and consider $\left(f_{n}, z_{n}\right)_{n}$, a special sequence for $H$ associated to $(\mathcal{I}, s, A)$. By (1.2), there exists $c>0$ such that

$$
\left\|\langle A\rangle^{-s} f_{n}\right\| \leq c\left\|\langle A\rangle^{s}\left(H-z_{n}\right) f_{n}\right\| .
$$


This implies $\eta=0$.

The previous result can be partially localized in energy.

Proposition 2.12. Let $(\mathcal{I}, s, A)$ be a triplet as in (1.2) with $0 \leq s<1$. Let $\mathcal{I}^{\prime \prime}$ be open such that $\overline{\mathcal{I}} \subset \mathcal{I}^{\prime \prime}$ and $H \in \mathcal{C}_{\mathcal{I}^{\prime \prime}}^{1}(A)$. Let $\theta \in \mathcal{C}_{c}^{\infty}(\mathbb{R})$ such that $\theta=1$ near $\mathcal{I}$. Let $\varphi: \mathbb{R} \rightarrow \mathbb{R}$ borelian such that, for $t \in \operatorname{supp} \theta$, $\varphi(t)=t$. Let $\left(f_{n}, z_{n}\right)_{n}$ be a special sequence for $H$ associated to $(\mathcal{I}, s, A)$ with mass $\eta$. Then, writing $\tilde{\theta}=1-\theta$,

(1) $\tilde{\theta}(H) f_{n}$ tends to 0 ,

(2) $\left(\theta(H) f_{n}, z_{n}\right)_{n}$ is a special sequence for $\varphi(H)$ associated to $(\mathcal{I}, s, A)$ with mass $\eta$.

Proof. Since

$$
\left\|\tilde{\theta}(H) f_{n}\right\| \leq\left\|\tilde{\theta}(H)\left(H-z_{n}\right)^{-1}\langle A\rangle^{-s}\right\| \cdot\left\|\langle A\rangle^{s}\left(H-z_{n}\right) f_{n}\right\|
$$

and since $t \mapsto \tilde{\theta}(t) /\left(t-z_{n}\right)$ is uniformly bounded in $n,\left\|\tilde{\theta}(H) f_{n}\right\|$ tends to 0 . Since $s \geq 0,\left\|\langle A\rangle^{-s} \tilde{\theta}(H) f_{n}\right\| \rightarrow 0$ and therefore $\left\|\langle A\rangle^{-s} \theta(H) f_{n}\right\| \rightarrow$ $\eta$. Since $H \in \mathcal{C}_{\mathcal{I}^{\prime \prime}}^{1}(A), \theta(H) \in \mathcal{C}^{1}(A)$. Since $s<1,\left\|\langle A\rangle^{s} \theta(H)\langle A\rangle^{-s}\right\|$ is bounded, by Proposition B.2. Now,

$$
\left\|\langle A\rangle^{s}\left(\varphi(H)-z_{n}\right) \theta(H) f_{n}\right\| \leq\left\|\langle A\rangle^{s} \theta(H)\langle A\rangle^{-s}\right\| \cdot\left\|\langle A\rangle^{s}\left(H-z_{n}\right) f_{n}\right\|
$$

which tends to 0.

Now we can perform the reduction to some $H_{\tau}$ (cf. (2.4)).

Proposition 2.13. Let $(\mathcal{I}, s, A)$ be a triplet as in (1.2) with $0 \leq s<1$. Let $\mathcal{I}^{\prime \prime}$ be open such that $\overline{\mathcal{I}} \subset \mathcal{I}^{\prime \prime}$ and $H \in \mathcal{C}_{\mathcal{I}^{\prime \prime}}^{1}(A)$. Let $\tau \in \mathcal{C}_{c}^{\infty}\left(\mathcal{I}^{\prime \prime}\right)$ such that $\tau=1$ near $\mathcal{I}$. If the LAP respectively to $(\mathcal{I}, s, A)$ holds true for $H_{\tau}$ then it holds true for $H$.

Proof. By contraposition, the result follows from Propositions 2.11 and 2.12

Remark 2.14. There is another proof of Proposition 2.13. Let $\theta \in$ $\mathcal{C}_{c}^{\infty}(\mathbb{R})$ with $\theta=1$ near $\mathcal{I}$ and $\tau \theta=\theta$. Then, using a Neumann serie for $|z|$ large enough and $z \notin \mathbb{R}$, we can show that $(H-z)^{-1} \theta(H)=$ $\left(H_{\tau}-z\right)^{-1} \theta(H)$. By analyticity, this holds true for $z \notin \mathbb{R}$. Therefore, if the LAP respectively to $(\mathcal{I}, s, A)$ is true for $H_{\tau}$ so is it for $H$, since $\langle A\rangle^{s} \theta(H)\langle A\rangle^{-s}$ is bounded. 
2.4. A Virial-like Theorem. In Remark 1.1, we recalled the Virial Theorem. Our approach is based on the following Virial-like result.

Proposition 2.15. Let $\left(f_{n}, z_{n}\right)_{n}$ be a special sequence for a bounded, self-adjoint operator $H_{b}$ respectively to $(\mathcal{I}, s, A)$, as in (1.2) with $s \geq 0$. For any bounded borelian function $\phi$,

$$
\lim _{n \rightarrow \infty}\left\langle f_{n},\left[H_{b}, \phi(A)\right] f_{n}\right\rangle=0 .
$$

Proof. Since $\left[H_{b}, \phi(A)\right]=\left[H_{b}-z_{n}, \phi(A)\right]$,

$$
\begin{aligned}
& \left\langle f_{n},\left[H_{b}, \phi(A)\right] f_{n}\right\rangle=2 i \operatorname{Im}\left(z_{n}\right)\left\langle f_{n}, \phi(A) f_{n}\right\rangle \\
& \quad+\left\langle\left(H_{b}-z_{n}\right) f_{n}, \phi(A) f_{n}\right\rangle+\left\langle\phi(A)^{*} f_{n},\left(H_{b}-z_{n}\right) f_{n}\right\rangle .
\end{aligned}
$$

By Definition 2.10, there exists $C>0$ such that

$$
\begin{aligned}
\left|\left\langle\left(H_{b}-z_{n}\right) f_{n}, \phi(A) f_{n}\right\rangle\right| & \leq\left|\left\langle\langle A\rangle^{s}\left(H_{b}-z_{n}\right) f_{n},\langle A\rangle^{-s} \phi(A) f_{n}\right\rangle\right| \\
& \leq C\|\phi(A)\| \cdot\left\|\langle A\rangle^{s}\left(H_{b}-z_{n}\right) f_{n}\right\| \underset{n \rightarrow \infty}{\rightarrow} 0 .
\end{aligned}
$$

Similarly, $\lim \left\langle\phi(A)^{*} f_{n},\left(H_{b}-z_{n}\right) f_{n}\right\rangle=0$. By Definition 2.10.

$$
\begin{aligned}
\operatorname{Im}\left(z_{n}\right) \cdot\left\|f_{n}\right\|^{2} & =\operatorname{Im}\left\langle f_{n},\left(H_{b}-z_{n}\right) f_{n}\right\rangle \\
& =\operatorname{Im}\left\langle\langle A\rangle^{-s} f_{n},\langle A\rangle^{s}\left(H_{b}-z_{n}\right) f_{n}\right\rangle \underset{n \rightarrow \infty}{\rightarrow} 0 .
\end{aligned}
$$

Since

$$
\left|\operatorname{Im}\left(z_{n}\right)\left\langle f_{n}, \phi(A) f_{n}\right\rangle\right| \leq\left|\operatorname{Im}\left(z_{n}\right)\right| \cdot\left\|f_{n}\right\|^{2} \cdot\|\phi(A)\|,
$$

we obtain the desired result.

Remark 2.16. If $H_{b}$ is not bounded, Proposition 2.15 works, provided the commutator $\left[H_{b}, \phi(A)\right]$ is considered as quadratic form.

2.5. Sketch of our proof and interpretation. To prove Theorem 2.7. we only need to show the LAP for $H_{\tau}$ on $\mathcal{I}^{\prime}$ by Proposition 2.13. In view of Proposition 2.11, we consider a special sequence $\left(f_{n}, z_{n}\right)_{n}$ for $H_{\tau}$ associated to the triplet $(\mathcal{I}, s, A)$, with $s>1 / 2$, and we show that $\eta=0$. By Remark 2.3. we may assume that $s \in] 1 / 2 ; 2 / 3\left[\right.$. For $R>1$, let $\chi_{R}+\tilde{\chi}_{R}=1$ be a smooth partition of unity on $\mathbb{R}$ with $\chi_{R}$ localized in $\{t \in \mathbb{R} ;|t| \leq 2 R\}$. It suffices to show that $\lim _{R \rightarrow \infty} \lim \sup _{n \rightarrow \infty}\left\|\tilde{\chi}_{R}(A)\langle A\rangle^{-s} f_{n}\right\|=0$ and $\lim _{R \rightarrow \infty} \lim \sup _{n \rightarrow \infty}\left\|\chi_{R}(A)\langle A\rangle^{-s} f_{n}\right\|=0$. From the strict Mourre estimate (2.6), we deduce (2.5) with $K=0$. We apply the latter to $\tilde{\chi}_{R}(A)\langle A\rangle^{-s} f_{n}$. After several commutations, the use of Proposition 2.15] and the use of the assumption $s>1 / 2$, we find some $\epsilon>0$ 
such that, for all $R>1$,

$$
\limsup _{n \rightarrow \infty}\left\|\tilde{\chi}_{R}(A)\langle A\rangle^{-s} f_{n}\right\|=O\left(R^{-\epsilon}\right) .
$$

Next we apply the Mourre estimate (2.5) to $\chi_{R}(A) f_{n}$. After several commutations, the use of Proposition 2.15, and the use of (2.8), we get $\lim _{R \rightarrow \infty} \lim \sup _{n \rightarrow \infty}\left\|\chi_{R}(A) f_{n}\right\|=0$. Since $s \geq 0$, we obtain the desired results yielding $\eta=0$.

This proof provides the following new interpretation of Theorems 1.2 and 2.7. The strict Mourre estimate excludes the existence of a special sequence of positive mass, yielding the LAP, in a similar way as it excludes the existence of a bound state in Remark 1.1. Our Virial-like Theorem plays the role of the usual Virial Theorem.

\section{A NEW PROOF OF THE LAP.}

Here we complete the proof of Theorem 2.7 sketched in Subsection 2.5. We assume the assumptions of Theorem 2.7 satisfied and take some interval $\mathcal{I}^{\prime} \subset \stackrel{\circ}{\mathcal{I}}$. Let $\theta \in \mathcal{C}_{c}^{\infty}(\mathcal{I})$ with $\theta=1$ on $\mathcal{I}^{\prime}$. Applying $\theta(H)$ on both sides, we deduce from (2.6) the strict Mourre estimate (2.5) (i.e. with $K=0)$. We consider a special sequence $\left(f_{n}, z_{n}\right)_{n}$ for $H_{\tau}$ associated to $(\mathcal{I}, s, A)$ with $s \in] 1 / 2 ; 2 / 3[$. By Proposition 2.12, we may assume that $\theta(H) f_{n}=f_{n}$, for all $n$. Let us fix some notation. Let $\chi \in \mathcal{C}_{c}^{\infty}(\mathbb{R})$ such that

$$
\chi=1 \text { on }[-1,1] \text { and } \chi=0 \text { on } \mathbb{R} \backslash[-2,2] .
$$

We shall require other properties satisfied by $\chi$ (see (3.14) below). For $R>1$, we set $\chi_{R}(x)=\chi(x / R)$ for all $x \in \mathbb{R}$ and $\tilde{\chi}_{R}=1-\chi_{R}$. We denote by $O_{R}(\cdot)$ (resp. $\left.o_{R}(\cdot)\right)$ the Landau symbol $O$ (resp. o) where the subscript $R$ means that the bound (resp. the limit) is uniform w.r.t. the other variables.

3.1. Proof of Theorem 2.7. Let $\chi \in \mathcal{C}_{c}^{\infty}(\mathbb{R})$ satisfying (3.9) and (3.14). From Proposition 3.6 and Corollary 3.2 below, we derive that, for all $\varepsilon>0$,

$$
\begin{aligned}
\eta & =\lim _{n \rightarrow \infty}\left\|\langle A\rangle^{-s} f_{n}\right\| \\
& \leq \limsup _{n \rightarrow \infty}\left(\left\|\tilde{\chi}_{R}(A)\langle A\rangle^{-s} f_{n}\right\|+\left\|\chi_{R}(A)\langle A\rangle^{-s} f_{n}\right\|\right) \\
& \leq \limsup _{n \rightarrow \infty}\left(\left\|\tilde{\chi}_{R}(A)\langle A\rangle^{-s} f_{n}\right\|+\left\|\chi_{R}(A) f_{n}\right\|\right)=O\left(R^{2 s-2+\varepsilon}\right) .
\end{aligned}
$$


Letting $R$ go to infinity, we obtain that $\eta=0$. By Proposition 2.11. the LAP holds true for $H_{\tau}$ respectively to $\left(\mathcal{I}^{\prime}, s, A\right)$.

3.2. A "large $|A|$ " estimate. We stress that, in this subsection, we suppose that $1 / 2<s<1$. The aim of this part is to show

Proposition 3.1. Let $\mathcal{I}^{\prime}$ be closed with $\mathcal{I}^{\prime} \subset \stackrel{\circ}{\mathcal{I}}$ and let $\left(f_{n}, z_{n}\right)_{n}$ be a special sequence for $H_{\tau}$ respectively to $\left(\mathcal{I}^{\prime}, s, A\right)$ with $1>s>1 / 2$. Assume that the Mourre estimate (2.5) holds true with $\theta=1$ on $\mathcal{I}^{\prime}$ and $K=0$. Let $\chi \in \mathcal{C}_{c}^{\infty}(\mathbb{R})$ satisfying (3.9) and (3.14) (below). Then, there exist $c^{\prime}>0, R_{1}>2$, and a family $\left(\phi_{R}\right)_{R>1}$ in $L^{\infty}(\mathbb{R})$, such that, for all $R \geq R_{1}$,

$$
\begin{aligned}
\left\langle f_{n},\left[H_{\tau}, i \phi_{R}(A)\right]\right. & \left.f_{n}\right\rangle \geq c^{\prime}\left\|\tilde{\chi}_{R}(A)\langle A\rangle^{-s} f_{n}\right\|^{2} \\
& +O_{R}\left(R^{-1}\right) \cdot\left\|\tilde{\chi}_{R}(A)\langle A\rangle^{-s} f_{n}\right\| \\
& +O_{R}\left(R^{2 s-2}\right) \cdot\left\|\tilde{\chi}_{R / 2}(A)\langle A\rangle^{-s} f_{n}\right\| .
\end{aligned}
$$

Corollary 3.2. Under the hypotheses of Proposition 3.1,

$$
\forall \alpha>2 s-2, \quad \limsup _{n \rightarrow \infty}\left\|\tilde{\chi}_{R}(A)\langle A\rangle^{-s} f_{n}\right\|=O\left(R^{\alpha}\right)
$$

Proof. Note first that, for $a>0, \varepsilon \geq 0$, and $b, c \in \mathbb{R}$,

$$
\varepsilon \geq a X^{2}+b X+c^{2} \Longrightarrow|X| \leq \sqrt{\varepsilon / a}+O(|b|+|c|),
$$

the latter term being independent of $\varepsilon$. Since $\left(\left\|\langle A\rangle^{-s} f_{n}\right\|\right)_{n}$ is bounded by Definition 2.10, it suffices to prove (3.11) for large $R$. For fixed $R \geq R_{1}$, we combine (3.10) with (3.12) and Proposition 2.15 to get

$$
\begin{aligned}
& \limsup _{n \rightarrow \infty}\left\|\tilde{\chi}_{R}(A)\langle A\rangle^{-s} f_{n}\right\| \leq O\left(R^{-1}\right) \\
& +O\left(R^{s-1}\right) \cdot \limsup _{n \rightarrow \infty}\left\|\tilde{\chi}_{R / 2}(A)\langle A\rangle^{-s} f_{n}\right\|^{1 / 2} .
\end{aligned}
$$

We use a bootstrapping argument. Since $\left(\left\|\langle A\rangle^{-s} f_{n}\right\|\right)_{n}$ is bounded, so is $\left(\left\|\tilde{\chi}_{R / 2}(A)\langle A\rangle^{-s} f_{n}\right\|\right)_{n}$. Then (3.13) gives (3.11) for $\alpha=\alpha_{0}=s-1$. Now we use this new estimate in (3.13) to get (3.11) for $\alpha=\alpha_{1}=$ $3(s-1) / 2$. By induction, we get (3.11) for a sequence $\left(\alpha_{n}\right)_{n}$ satisfying $\alpha_{n+1}=\alpha_{n} / 2+(s-1)$, for all $n \in \mathbb{N}$. By a fixed point argument, $\alpha_{n} \rightarrow 2(s-1)$. This yields the result.

Our strategy to prove Proposition 3.1 is the following. We apply the strict Mourre estimate (2.5) (with $K=0$ ) to $\tilde{\chi}_{R}(A)\langle A\rangle^{-s} f_{n}$. We move the $\theta(H)$ to the $f_{n}$, which absorb them, since $\theta(H) f_{n}=f_{n}$. We want to pull the weights $\tilde{\chi}_{R}(A)\langle A\rangle^{-s}$ into the commutator $\left[H_{\tau}, A\right]$, in order to get the term on the l.h.s of (3.10) with $\phi_{R}(t)=t\langle t\rangle^{-2 s} \tilde{\chi}_{R}(t)^{2}$. In 
view of the proof of Corollary 3.2 , we need $2 s \geq 1$. Our manipulation produces of course error terms which should be small. Using $s>1 / 2$, we actually prove this smallness if we only move the $\theta(H)$ and the $\langle A\rangle^{-s}$. Choosing appropriate functions $\phi_{R}$, we can move the $\tilde{\chi}_{R}(A)$ into the commutator producing an error term which has the good sign, up to small enough terms. To this end, we choose more carefully the function $\chi$ in (3.9). We demand that $\chi$ satisfies (3.9) and that

$$
\tilde{\chi}:=1-\chi=\tilde{\chi}_{+}+\tilde{\chi}_{-},
$$

where $\tilde{\chi} 1_{\mathbb{R}^{\sigma}}=\tilde{\chi}_{\sigma}$ for $\sigma \in\{-,+\}$, such that $\tilde{\chi}_{\sigma}$ and $\sigma \tilde{\chi}_{\sigma}^{\prime}$ are square of some smooth functions (see for instance the appendix in DG for their existence). Let $R>1$. We set $\tilde{\chi}_{\sigma, R}=\tilde{\chi}_{\sigma}(t / R)$. Notice that $\tilde{\chi}_{R}=\tilde{\chi}_{+, R}+\tilde{\chi}_{-, R}$ and $\tilde{\chi}_{R}^{2}=\tilde{\chi}_{+, R}^{2}+\tilde{\chi}_{-, R}^{2}$.

Proof of Proposition 3.1. Let

$$
h>\sup _{t \in \mathbb{R}}|t|\langle t\rangle^{-2 s} .
$$

Let $R>1$ and $\phi_{R} \in \mathcal{C}^{\infty}(\mathbb{R})$ defined by

$$
\phi_{R}(t)=\sum_{\sigma \in\{-,+\}} \tilde{\chi}_{\sigma, R}^{2}(t)\left(\sigma h-t\langle t\rangle^{-2 s}\right) .
$$

For all $f \in \mathscr{H}$,

$$
\begin{aligned}
& \left\langle f,\left[H_{\tau}, i \phi_{R}(A)\right] f\right\rangle=\left\langle f, \tilde{\chi}_{R}(A)\left[H_{\tau},-i A\langle A\rangle^{-2 s}\right] \tilde{\chi}_{R}(A) f\right\rangle \\
& (3.17)+2 \sum_{\sigma \in\{-,+\}} \operatorname{Re}\left\langle f,\left(\sigma h-A\langle A\rangle^{-2 s}\right) \tilde{\chi}_{\sigma, R}(A)\left[H_{\tau}, i \tilde{\chi}_{\sigma, R}(A)\right] f\right\rangle .
\end{aligned}
$$

We can find some $R_{1}>2$ (see Lemma 3.4 below) such that, for $R \geq R_{1}$,

$$
\left\langle\tilde{\chi}_{R}(A) f_{n},\left[H_{\tau},-i A\langle A\rangle^{-2 s}\right] \tilde{\chi}_{R}(A) f_{n}\right\rangle \geq c^{\prime}\left\|\tilde{\chi}_{R}(A)\langle A\rangle^{-s} f_{n}\right\|^{2}
$$

with $c^{\prime}=(2 s-1)^{-1} c / 2>0$. Since we are not able to estimate properly the second term on the r.h.s of (3.17), we indend to use some positivity argument to get rid of it. In view of (B.4), we choosed $\phi_{R}$ in (3.16) such that, the function $\psi_{R} \in \mathcal{C}_{c}^{\infty}(\mathbb{R})$ defined by

$$
\begin{aligned}
\psi_{R}(t) & =R\left(\phi_{R}^{\prime}(t)-\tilde{\chi}_{R}^{2}(t)(d / d t)\left(-t\langle t\rangle^{-2 s}\right)\right) \\
& =\sum_{\sigma \in\{-,+\}}\left(\tilde{\chi}_{\sigma}^{\prime}\right)_{R}(t)\left(\sigma h-t\langle t\rangle^{-2 s}\right) \tilde{\chi}_{\sigma, R}(t)
\end{aligned}
$$

is the square of a smooth function. We put a factor $R$ in front to ensure that the family $\left(\psi_{R}\right)_{R}$ is bounded in some symbol space (see 
Lemma A.4). Notice that $\operatorname{supp} \psi_{R} \subset[-2 R,-R] \cup[R, 2 R]$. We define

$$
C_{R}:=\psi_{R}^{1 / 2}(A) \text { and note that } C_{R} \tilde{\chi}_{R / 2}(A)=C_{R} .
$$

We can show (see Lemma 3.5 below) that

$$
\left\langle C_{R} f_{n},\left[H_{\tau}, i A\right] C_{R} f_{n}\right\rangle \geq O_{R}\left(R^{2 s-1}\right) \cdot\left\|\tilde{\chi}_{R / 2}(A)\langle A\rangle^{-s} f_{n}\right\| .
$$

By Lemma C.4

$$
\left\langle C_{R}^{2} f_{n},\left[H_{\tau}, i A\right] f_{n}\right\rangle \geq O_{R}\left(R^{2 s-1}\right) \cdot\left\|\tilde{\chi}_{R / 2}(A)\langle A\rangle^{-s} f_{n}\right\|,
$$

since $\left\|\tilde{\chi}_{R / 2}(A)\langle A\rangle^{-s} f_{n}\right\|=O_{R}\left(R^{0}\right)$. Now, by Lemma C.2,

$$
\begin{aligned}
\left\langle f_{n},\left(\sigma h-A\langle A\rangle^{-2 s}\right) \tilde{\chi}_{\sigma, R}(A)\left[H_{\tau}, \tilde{\chi}_{\sigma, R}(A)\right] f_{n}\right\rangle \geq & \geq \\
+ & O_{R}\left(R^{2 s-2}\right) \cdot\left\|\tilde{\chi}_{R / 2}(A)\langle A\rangle^{-s} f_{n}\right\| .
\end{aligned}
$$

This yields, together with (3.17) and (3.18), the result.

To prove (3.18) and (3.21), we need the following lemmata.

Lemma 3.3. Under the assumptions of Proposition 3.1,

$$
\begin{gathered}
\left\|\left[\theta(H), \chi_{R}(A)\right] f_{n}\right\|=O_{R}\left(R^{s-1}\right),\left\|\left[\theta(H), C_{R}\right] f_{n}\right\|=O_{R}\left(R^{s-1}\right), \\
\left\|\left[\theta(H),\langle A\rangle^{-s} \tilde{\chi}_{R}(A)\right] f_{n}\right\|=O_{R}\left(R^{-1}\right) .
\end{gathered}
$$

Proof. By Corollary A.3, the families $\left(\tilde{\chi}_{R}\right)_{R>1}$ and $\left(\psi_{R}^{1 / 2}\right)_{R>1}$ are bounded in $\mathcal{S}^{0}$, while the family $\left(\sigma_{R}: t \mapsto\langle t\rangle^{-s} \tilde{\chi}_{R}(t)\right)_{R>1}$ is bounded in $\mathcal{S}^{-s}$. Furthermore, ] $-R, R$ [ does not intersect the supports of $\tilde{\chi}_{R}$, $\psi_{R}^{1 / 2}$, and $\sigma_{R}$. By Lemma B.3,

$$
\begin{array}{r}
\left\|\left[\theta(H), \chi_{R}(A)\right]\langle A\rangle^{s}\right\|=\left\|\left[\theta(H), \tilde{\chi}_{R}(A)\right]\langle A\rangle^{s}\right\|=O\left(R^{s-1}\right), \\
\left\|\left[\theta(H), C_{R}\right]\langle A\rangle^{s}\right\|=O\left(R^{s-1}\right),\left\|\left[\theta(H),\langle A\rangle^{-s} \tilde{\chi}_{R}(A)\right]\langle A\rangle^{s}\right\|=O\left(R^{-1}\right) .
\end{array}
$$

Using the boundness of $\left(\left\|\langle A\rangle^{-s} f_{n}\right\|\right)_{n}$ (cf. Definition 2.10), this yields the results.

Lemma 3.4. The inequality (3.18) holds true.

Proof. Applying (2.5) (with $K=0)$ to the $\tilde{\chi}_{R}(A)\langle A\rangle^{-s} f_{n}$,

$$
\begin{aligned}
\left\langle\theta(H) \tilde{\chi}_{R}(A)\langle A\rangle^{-s} f_{n},\left[H_{\tau}, i A\right] \theta(H) \tilde{\chi}_{R}(A)\langle A\rangle^{-s} f_{n}\right\rangle \\
\geq \quad c\left\|\theta(H) \tilde{\chi}_{R}(A)\langle A\rangle^{-s} f_{n}\right\|^{2} .
\end{aligned}
$$

Recall that $\theta(H) f_{n}=f_{n}$. By Lemma 3.3.

$$
\left\{\begin{array}{l}
\left|\left\langle\left[\theta(H), \tilde{\chi}_{R}(A)\langle A\rangle^{-s}\right] f_{n},\left[H_{\tau}, i A\right] \tilde{\chi}_{R}(A)\langle A\rangle^{-s} f_{n}\right\rangle\right|, \\
\left|\left\langle\left[\theta(H), \tilde{\chi}_{R}(A)\langle A\rangle^{-s}\right] f_{n},\left[H_{\tau}, i A\right] \theta(H) \tilde{\chi}_{R}(A)\langle A\rangle^{-s} f_{n}\right\rangle\right|, \\
\left|\left\langle\left[\theta(H), \tilde{\chi}_{R}(A)\langle A\rangle^{-s}\right] f_{n}, \tilde{\chi}_{R}(A)\langle A\rangle^{-s} f_{n}\right\rangle\right| \\
\left|\left\langle\left[\theta(H), \tilde{\chi}_{R}(A)\langle A\rangle^{-s}\right] f_{n}, \theta(H) \tilde{\chi}_{R}(A)\langle A\rangle^{-s} f_{n}\right\rangle\right|
\end{array}\right.
$$


are bounded above by $O_{R}\left(R^{-1}\right) \cdot\left\|\tilde{\chi}_{R}(A)\langle A\rangle^{-s} f_{n}\right\|$. Therefore,

$$
\begin{aligned}
\left\langle\tilde{\chi}_{R}(A)\right. & \left.\langle A\rangle^{-s} f_{n},\left[H_{\tau}, i A\right] \tilde{\chi}_{R}(A)\langle A\rangle^{-s} f_{n}\right\rangle \\
& \geq c\left\|\tilde{\chi}_{R}(A)\langle A\rangle^{-s} f_{n}\right\|^{2}+O_{R}\left(R^{-1}\right) \cdot\left\|\tilde{\chi}_{R}(A)\langle A\rangle^{-s} f_{n}\right\|
\end{aligned}
$$

By Lemma C.1. for $c^{\prime}=(2 s-1)^{-1} c / 2$, (3.18) holds true for $R \geq R_{1}>2$, if $R_{1}$ is large enough.

Lemma 3.5. The inequality (3.21) holds true.

Proof. From (2.5) (with $K=0$ ) applied to the $C_{R} f_{n}$, where $C_{R}$ is defined in (3.20), we derive that

$$
\left\langle\theta(H) C_{R} f_{n},\left[H_{\tau}, i A\right] \theta(H) C_{R} f_{n}\right\rangle \geq 0 .
$$

Thanks to (3.20) and to the Lemmata 3.3 and C.3.

$$
\left\{\begin{array}{l}
\left|\left\langle\left[\theta(H), C_{R}\right] f_{n},\left[H_{\tau}, i A\right] C_{R}\langle A\rangle^{s} \tilde{\chi}_{R / 2}(A)\langle A\rangle^{-s} f_{n}\right\rangle\right|, \\
\left|\left\langle\left[\theta(H), C_{R}\right] f_{n},\left[H_{\tau}, i A\right] \theta(H) C_{R}\langle A\rangle^{s} \tilde{\chi}_{R / 2}(A)\langle A\rangle^{-s} f_{n}\right\rangle\right|
\end{array}\right.
$$

are bounded by $O_{R}\left(R^{2 s-1}\right) \cdot\left\|\tilde{\chi}_{R / 2}(A)\langle A\rangle^{-s} f_{n}\right\|$, yielding (3.21).

3.3. Absence of mass. The aim of this part is to show

Proposition 3.6. Under the hypotheses of Proposition 3.1 with $1 / 2<$ $s<2 / 3$,

$$
\lim _{R \rightarrow \infty} \limsup _{n \rightarrow \infty}\left\|\chi_{R}(A) f_{n}\right\|=0
$$

Proof. Applying (2.5) (with $K=0$ ) to the $\chi_{R}(A) f_{n}$,

$$
\left\langle\chi_{R}(A) f_{n}, \theta(H)\left[H_{\tau}, i A\right] \theta(H) \chi_{R}(A) f_{n}\right\rangle \geq c\left\|\theta(H) \chi_{R}(A) f_{n}\right\|^{2} .
$$

By Lemma 3.3.

$$
\left\{\begin{array}{l}
\left|\left\langle\left[\theta(H), \chi_{R}(A)\right] f_{n},\left[H_{\tau}, i A\right] \chi_{R}(A) f_{n}\right\rangle\right| \\
\left|\left\langle\left[\theta(H), \chi_{R}(A)\right] f_{n},\left[H_{\tau}, i A\right] \theta(H) \chi_{R}(A) f_{n}\right\rangle\right| \\
\left|\left\langle\left[\theta(H), \chi_{R}(A)\right] f_{n}, \chi_{R}(A) f_{n}\right\rangle\right| \\
\left|\left\langle\left[\theta(H), \chi_{R}(A)\right] f_{n}, \theta(H) \chi_{R}(A) f_{n}\right\rangle\right|
\end{array}\right.
$$

are bounded by $O_{R}\left(R^{s-1}\right) \cdot\left\|\chi_{R}(A) f_{n}\right\|$. Therefore, $\left\langle\chi_{R}(A) f_{n},\left[H_{\tau}, i A\right] \chi_{R}(A) f_{n}\right\rangle \geq c\left\|\chi_{R}(A) f_{n}\right\|^{2}+O_{R}\left(R^{s-1}\right) \cdot\left\|\chi_{R}(A) f_{n}\right\|$.

Since $s<2 / 3$, we can find $\beta>0$ (see Lemma 3.7 below) such that

$$
\left|\left\langle\left[H_{\tau}, \chi_{R}(A)\right] f_{n}, i A \chi_{R}(A) f_{n}\right\rangle\right| \leq O_{R}\left(R^{-\beta}\right)\left\|\chi_{R}(A) f_{n}\right\| .
$$

This yields

$$
\left\langle f_{n},\left[H_{\tau}, i A \chi_{R}^{2}(A)\right] f_{n}\right\rangle \geq c\left\|\chi_{R}(A) f_{n}\right\|^{2}+o_{R}(1) \cdot\left\|\chi_{R}(A) f_{n}\right\| .
$$


Now, we combine (3.12) and Proposition 2.15 to arrive at

$$
\limsup _{n \rightarrow \infty}\left\|\chi_{R}(A) f_{n}\right\|=o_{R}(1) .
$$

To complete the proof of Proposition 3.6, we show

Lemma 3.7. Under the assumptions of Proposition 3.6, there exists $\beta>0$ such that (3.23) holds true.

Proof. We decompose $\left\langle\left[H_{\tau}, \chi_{R}(A)\right] f_{n}, i A \chi_{R}(A) f_{n}\right\rangle$ as

$$
\begin{array}{r}
\quad\left\langle\left[H_{\tau}, \tilde{\chi}_{R}(A)\right] \tilde{\chi}_{R / 2}(A) f_{n}, i A \chi_{R}(A) f_{n}\right\rangle \\
+\left\langle\left[H_{\tau}, \tilde{\chi}_{R}(A)\right] \chi_{R / 2}(A) f_{n}, i A \chi_{R}(A) f_{n}\right\rangle
\end{array}
$$

Since $\left(\tilde{\chi}_{R}\right)_{R}$ is bounded in $\mathcal{S}^{0}$ (cf. Corollary A.3) and since the support of $\tilde{\chi}_{R}$ does not intersect ] $-R, R$, Lemma B.3 for $k=1$ ensures that $A\left[H_{\tau}, \tilde{\chi}_{R}(A)\right]\langle A\rangle^{s}$ is bounded and its norm is $O\left(R^{s}\right)$. Since $s<2 / 3$, we can find $\alpha \in]-s, 2 s-2[$. This implies, using Corollary 3.2. that the absolute value of (3.24) is bounded by $O_{R}\left(R^{s+\alpha}\right) \cdot\left\|\chi_{R}(A) f_{n}\right\|$, with $s+\alpha<0$. By Proposition B.2 with $k=2$,

$\left[H_{\tau}, \tilde{\chi}_{R}(A)\right] \chi_{R / 2}(A)=\left[H_{\tau}, A\right] \tilde{\chi}_{R}^{\prime}(A) \chi_{R / 2}(A)+I_{2} \chi_{R / 2}(A)=I_{2} \chi_{R / 2}(A)$ since $\operatorname{supp} \tilde{\chi}_{R}^{\prime} \cap \operatorname{supp} \tilde{\chi}_{R / 2}=\emptyset$. Lemma B.3 for $k=2$ implies that $A I_{2} \chi_{R / 2}(A)\langle A\rangle^{s}$ is bounded and its norm is $O\left(R^{s-1}\right)$. In particular, the absolute value of (3.25) is bounded by $O\left(R^{s-1}\right) \cdot\left\|\chi_{R}(A) f_{n}\right\|$.

\section{The LAP FOR THE REDUCED RESOLVENT.}

4.1. Motivation. An interesting consequence of the LAP (1.2) is the following propagation estimate (cf. Kato's local smoothness in ABG, JMP, [RS4]): there exists $C>0$ such that, for all $f \in \mathscr{H}$,

$$
\int_{-\infty}^{\infty}\left\|\langle A\rangle^{-s} e^{i t H} E_{\mathcal{I}}(H) f\right\|^{2} d t \leq C\|f\|^{2} .
$$

For $E_{\mathcal{I}}(H) f \neq 0$, the state $e^{i t H} E_{\mathcal{I}}(H) f$ must move to "regions where $|A|$ is large" when $t \rightarrow-\infty$ and $t \rightarrow+\infty$, since the integral converges. If $H f=\lambda f$ with $\lambda \in \mathcal{I}$ and $f \neq 0$, then $e^{i t H} E_{\mathcal{I}}(H) f=e^{i t \lambda} f$, $\left\|\langle A\rangle^{-s} e^{i t H} E_{\mathcal{I}}(H) f\right\|=\left\|\langle A\rangle^{-s} f\right\|$, and the integral in (4.26) diverges. Therefore, the LAP cannot hold true near an eigenvalue. However it is interesting to find out whether the estimate (4.26) holds true for nonzero states $E_{\mathcal{I}}(H) f$ which are orthogonal to the eigenvectors associated to eigenvalues in $\mathcal{I}$, i.e. nonzero states $P^{\perp} E_{\mathcal{I}}(H) f$. Now the 
reduced LAP (1.3) on $\mathcal{I}^{\prime}$ with $\mathcal{I} \subset \dot{\mathcal{I}}^{\prime}$ implies that

$$
\sup _{\operatorname{Re} z \in \mathcal{I}, \operatorname{Im} z \neq 0}\left\|\langle A\rangle^{-s}(H-z)^{-1} P^{\perp} E_{\mathcal{I}^{\prime}}(H)\langle A\rangle^{-s}\right\|<\infty
$$

since $(H-z)^{-1} E_{\mathbb{R} \backslash \mathcal{I}^{\prime}}(H)$ is uniformly bounded, yielding (4.26) with $f$ replaced by $P^{\perp} f$ by Kato's local smoothness (cf. [ABG, RS4]). Theorem 1.4 gives a situation where the latter estimate holds true.

4.2. Eigenvectors' regularity. Here we extend the result of [C] on the regularity w.r.t. $A$ of eigenvectors of $H$.

Proposition 4.1. Let $\mathcal{I}$ be a bounded, open interval that is included in the continuous spectrum of $H$. Let $\mathcal{I}^{\prime \prime}$ be an open interval such $\overline{\mathcal{I}} \subset \mathcal{I}^{\prime \prime}$. Let $H \in \mathcal{C}_{\mathcal{I}^{\prime \prime}}^{k}(A)$ with integer $k \geq 2$. Assume that the Mourre estimate (1.1) holds true on $\mathcal{I}$. Then, for any eigenvector $f$ of $H$ such that $E_{\mathcal{I}}(H) f=f, f \in \mathcal{D}\left(A^{k-2}\right)$.

Proof. Let $\tau \in \mathcal{C}_{c}^{\infty}\left(\mathcal{I}^{\prime \prime}\right)$ such that $\tau=1$ near $\mathcal{I}$. Take $H_{\tau}$ as in (2.4). Let $f$ be an eigenvector of $H$ such that $E_{\mathcal{I}}(H) f=f$. It is also an eigenvector of $H_{\tau}$ with same eigenvalue. As in the proof of Lemma 2.5. we may replace $H$ par $H_{\tau}$ in the commutator in (1.1). Now, we can follow the proof in $\left[\mathrm{C}\right.$, since $H_{\tau} \in \mathcal{C}^{k}(A)$.

Remark 4.2. Proposition 4.1 extends the result in [C] since we only assume the "local" regularity $H \in \mathcal{C}_{\mathcal{I}^{\prime \prime}}^{k}(A)$.

4.3. Proof of Theorem 1.4. As in the proof of Theorem 1.2 in Subsection [2.2, $H \in \mathcal{C}_{\mathcal{I}^{\prime \prime}}^{2}(A)$, for any open interval $\mathcal{I}^{\prime \prime}$ with $\overline{\mathcal{I}} \subset \mathcal{I}^{\prime \prime}$. Let $\theta \in \mathcal{C}_{c}^{\infty}(\mathcal{I})$. In particular, $\theta(H) \in \mathcal{C}^{2}(A)$. By Remark 1.1. (1.1) implies that $P E_{\mathcal{I}}(H)$ is a finite dimensional projection. By Proposition 2.1. the hypothesis $\operatorname{Ran} P E_{\mathcal{I}}(H) \subset \mathcal{D}\left(A^{2}\right)$ implies that $P E_{\mathcal{I}}(H) \in \mathcal{C}^{2}(A)$ Since $\theta(H) P=\theta(H) P E_{\mathcal{I}}(H), \theta(H) P \in \mathcal{C}^{2}(A)$. Let $\tau \in \mathcal{C}_{c}^{\infty}\left(\mathcal{I}^{\prime \prime}\right)$ such that $\tau=1$ near $\mathcal{I}$. Let $\mathcal{I}^{\prime}$ be closed with $\mathcal{I}^{\prime} \subset \stackrel{\circ}{\mathcal{I}}$ and $\theta \in \mathcal{C}_{c}^{\infty}(\mathcal{I})$ with $\theta=1$ near $\mathcal{I}^{\prime}$. By Lemma 2.5, (2.5) holds true (with $H_{\tau}$ defined in (2.4) ). Thus

$$
\begin{aligned}
P^{\perp} \theta(H)\left[H_{\tau}, i A\right] \theta(H) P^{\perp} \geq & c\left(\theta(H) P^{\perp}\right)^{2} \\
& +\theta(H) P^{\perp} K \theta(H) P^{\perp} .
\end{aligned}
$$

Let $\theta_{1} \in \mathcal{C}_{c}^{\infty}\left(\mathcal{I}^{\prime}\right)$. Since $P^{\perp}: 1-P$ projects onto the continuous spectral subspace of $H, \theta_{1}(H) P^{\perp}$ converges strongly to 0 as the support of $\theta_{1}$ shrinks to a point. Since $K$ compact, $\left\|K \theta_{1}(H) P^{\perp}\right\|$ goes to 0 in the 
same limit. Multiplying (4.27) by $\theta_{1}(H)$ on both sides and taking the support of $\theta_{1}$ small enough inside $\mathcal{I}^{\prime}$, we obtain

$$
P^{\perp} \theta_{1}(H)\left[H_{\tau}, i A\right] \theta_{1}(H) P^{\perp} \geq(c / 2)\left(\theta_{1}(H) P^{\perp}\right)^{2} .
$$

Around any point of $\mathcal{I}^{\prime}$, we thus can find some infinite interval $\mathcal{I}_{1} \subset \mathcal{I}$ such that the projected Mourre estimate (4.28) below holds true on $\mathcal{I}_{1}$. By Theorem 4.3, the reduced LAP holds true on any closed $\mathcal{I}_{1}^{\prime}$ with $\mathcal{I}_{1}^{\prime} \subset \stackrel{\circ}{\mathcal{I}}_{1}$. By compacity of $\mathcal{I}^{\prime}$, we get the reduced LAP on it.

So the proof of Theorem 1.4 reduces to the proof of a local and stronger version of it, namely

Theorem 4.3. Let $\mathcal{I}$ be a bounded, open interval. Let $\mathcal{I}^{\prime \prime}$ be an open interval such $\overline{\mathcal{I}} \subset \mathcal{I}^{\prime \prime}$. Let $H \in \mathcal{C}_{\mathcal{I}^{\prime \prime}}^{2}(A)$ and assume that, for all $\theta \in$ $\mathcal{C}_{c}^{\infty}(\mathcal{I}), \theta(H) P \in \mathcal{C}^{2}(A)$. Let $\tau \in \mathcal{C}_{c}^{\infty}\left(\mathcal{I}^{\prime \prime}\right)$ such that $\tau=1$ near $\mathcal{I}$. Assume the projected Mourre estimate

$$
P^{\perp} E_{\mathcal{I}}(H)\left[H_{\tau}, i A\right] E_{\mathcal{I}}(H) P^{\perp} \geq c E_{\mathcal{I}}(H) P^{\perp}, \text { with } c>0,
$$

holds true. Then, for any $s>1 / 2$ and any compact interval $\mathcal{I}^{\prime}$ with

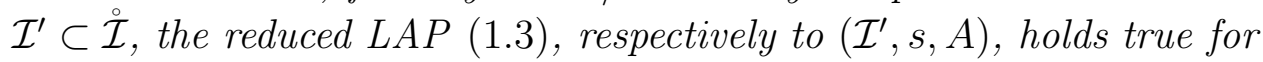
$H_{\tau}$ and $H$.

4.4. Proofs of Theorem 4.3. We shall give two proofs of Theorem 4.3. The first one is a direct generalization to the present context of our proof of Theorem 2.7. The second proof is close to the corresponding proof in $\mathrm{CGH}$ and shows that Theorem 2.7 actually applies to $H P^{\perp}$. In Remark 4.5, we compare the two proofs. In Remark 4.6. we comment on Sahbani's result (cf. [S] ) in this context.

First proof of Theorem 4.3. By Remark 2.3. we may assume that $1 / 2<s<1$. Assume the reduced LAP for $H$ false on some $\mathcal{I}^{\prime} \subset \dot{\mathcal{I}}$. Let $\theta \in \mathcal{C}_{c}^{\infty}(\mathcal{I})$ with $\theta=1$ on $\mathcal{I}^{\prime}$. Notice that, since $\theta(H), \theta(H) P \in \mathcal{C}^{2}(A)$, $\theta(H) P^{\perp} \in \mathcal{C}^{2}(A)$. Then, using the proof of Proposition 2.11 and 2.12. we can find a special sequence $\left(f_{n}, z_{n}\right)_{n}$ for $H_{\tau}$ with positive mass such that $\theta(H) f_{n}=f_{n}=P^{\perp} f_{n}$, for all $n$. Since

$$
\langle A\rangle^{-s} f_{n}=\langle A\rangle^{-s}\left(H_{\tau}-z_{n}\right)^{-1} P^{\perp}\langle A\rangle^{-s}\langle A\rangle^{s}\left(H_{\tau}-z_{n}\right) f_{n},
$$

the reduced LAP for $H_{\tau}$ on $\mathcal{I}^{\prime}$ must be false. So it suffices to prove the reduced LAP for $H_{\tau}$ on $\mathcal{I}^{\prime}$. Using Proposition 2.11] and 2.12 in a similar way, we can show that the reduced LAP for $H_{\tau}$ on $\mathcal{I}^{\prime}$ holds true if and only if, for all special sequence $\left(f_{n}, z_{n}\right)_{n}$ for $H_{\tau}$ such that $\theta(H) f_{n}=f_{n}=P^{\perp} f_{n}$, for all $n$, its mass is 0 . Now, we take such a 
special sequence $\left(f_{n}, z_{n}\right)_{n}$. Multiplying (4.28) on both sides by $\theta(H)$,

$$
P^{\perp} \theta(H)\left[H_{\tau}, i A\right] \theta(H) P^{\perp} \geq c\left(\theta(H) P^{\perp}\right)^{2} .
$$

Since $\theta(H) P^{\perp} \in \mathcal{C}^{2}(A)$, we can follow our proof of Theorem 2.7 in Section 3, yielding the reduced LAP for $H_{\tau}$ on $\mathcal{I}^{\prime}$.

Second proof of Theorem 4.3. Assume for a while that Theorem 4.3 holds true if $0 \notin \overline{\mathcal{I}}$. Under the assumptions of Theorem 4.3, we can find some real $\mu$ such that $0 \notin \mu+\overline{\mathcal{I}}$. Notice that $H$ and $H+\mu$ have the same eigenvalues and eigenvectors and that the eigenvalues of $H$ in $\mathcal{I}$ are the eigenvalues of $H+\mu$ in $\mu+\mathcal{I}$. For any $\varphi: \mathbb{R} \rightarrow \mathbb{R}$ bounded and borelian, $\varphi(H)=\varphi((H+\mu)-\mu)$, a function of $H+\mu$. Thus the assumptions of Theorem 4.3 are satisfied if $H$ is replaced by $H+\mu$ and $\mathcal{I}$ by $\mu+\mathcal{I}$, and $0 \notin \mu+\overline{\mathcal{I}}$. Thus, it suffices to prove it when $0 \notin \overline{\mathcal{I}}$.

For any $\theta \in \mathcal{C}^{\infty}(\mathbb{R} \backslash\{0\}), \theta(H) P^{\perp}=\theta\left(H P^{\perp}\right)$ by Lemma 4.4 below. Thus $H P^{\perp} \in \mathcal{C}_{\mathcal{I}^{\prime \prime}}^{2}(A)$. Furthermore, using Lemma 2.5] we derive from (4.28) the estimate, for $\theta \in \mathcal{C}_{c}^{\infty}(\mathcal{I})$ with $\theta=1$ near $\mathcal{I}^{\prime}$,

$$
\theta\left(H P^{\perp}\right)\left[\left(H P^{\perp}\right)_{\tau}, i A\right] \theta\left(H P^{\perp}\right) \geq c \theta^{2}\left(H P^{\perp}\right) .
$$

Now, we can apply Theorem 2.7 to $H P^{\perp}$ with $\mathcal{I}=\theta^{-1}(1)$, yielding the LAP for $H P^{\perp}$ on $\mathcal{I}^{\prime}$. Let $z \in \mathbb{C}$ with $\operatorname{Im}(z) \neq 0$. By Feshbach decomposition (see [BFS] for instance), $\left(H P^{\perp}-z\right)^{-1} P^{\perp}=(H-z)^{-1} P^{\perp}$. Let $\operatorname{Re}(z) \in \mathcal{I}^{\prime}$ and $s \in[0 ; 1[$. Setting $\tilde{\theta}=1-\theta$, we write

$$
\begin{array}{r}
\langle A\rangle^{-s}(H-z)^{-1} P^{\perp}\langle A\rangle^{-s}=\langle A\rangle^{-s}(H-z)^{-1} P^{\perp} \tilde{\theta}(H)\langle A\rangle^{-s} \\
+\langle A\rangle^{-s}\left(H P^{\perp}-z\right)^{-1}\langle A\rangle^{-s} \cdot\langle A\rangle^{s} \theta(H) P^{\perp}\langle A\rangle^{-s} .
\end{array}
$$

Since $\theta(H) P^{\perp} \in \mathcal{C}^{1}(A),\langle A\rangle^{s} \theta(H) P^{\perp}\langle A\rangle^{-s}$ is bounded by Proposition B.2. This yieds the reduced LAP (1.3) for $H$, since $(H-z)^{-1} \tilde{\theta}(H)$ is uniformly bounded for $\operatorname{Re}(z) \in \mathcal{I}^{\prime}$.

The second proof of Theorem 4.3 uses the following consequence of the Feshbach decomposition (see [BFS] for instance).

Lemma 4.4. For all $\varphi \in \mathcal{C}_{c}^{\infty}(\mathbb{R} \backslash\{0\}), \varphi\left(H P^{\perp}\right) P=0$ and $\varphi\left(H P^{\perp}\right)=$ $\varphi(H) P^{\perp}$.

Proof. Let $z \in \mathbb{C}$ with $\operatorname{Im}(z) \neq 0$. By Feshbach decomposition, $\left(H P^{\perp}-\right.$ $z)^{-1} P^{\perp}=(H-z)^{-1} P^{\perp}$. Using (B.1), $\varphi\left(H P^{\perp}\right) P^{\perp}=\varphi(H) P^{\perp}$. Since $\operatorname{Ran} P$ is contained in the kernel of $H P^{\perp}, \varphi\left(H P^{\perp}\right) P=\varphi(0) P=0$, by assumption on $\varphi$. Finally, $\varphi(H) P^{\perp}=\varphi\left(H P^{\perp}\right)-0$.

Remark 4.5. In the second proof, the idea is to replace $H$ par $H P^{\perp}$. Since we push that way the eigenvectors of $H$ leaving in $\operatorname{Ran} E_{\mathcal{I}}(H)$ in the kernel of $H P^{\perp}$, they are no longer an obstacle to the strict Mourre 
estimate on $\mathcal{I}$, if $0 \notin \overline{\mathcal{I}}$. The main difference between the two previous proofs is probably the use of energy translation for $H$ in the second one to avoid the case where $0 \in \overline{\mathcal{I}}$.

Remark 4.6. In the second proof, $H P^{\perp} \in \mathcal{C}_{\mathcal{I}}^{2}$, $(A)$ and (4.30) can be written as a Mourre estimate for $H P^{\perp}$. Under the assumptions of Theorem 4.3, Sahbani's result in [S] applies and the boundary values of the reduced resolvent have some Hölder continuity. As shown in the proof of Theorem 1.4, the assumption " $\theta(H) P \in \mathcal{C}^{2}(A)$ " is satisfied if the Mourre estimate (1.1) holds true on $\mathcal{I}$, included in the continuous spectrum of $H$, and if $H \in \mathcal{C}_{\mathcal{I}}^{4},(A)$, by Proposition 4.1

4.5. An artificial but instructive example. In this section, we construct an example of operators $H$ and $A$, for which Theorems 1.4 and 4.3 apply but the Mourre estimate (1.1) cannot be true. In particular, Theorems 1.2 and 2.7] do not apply to this example. Our contruction is quite artificial but our operators $H$ and $A$ presents some structural similarity with operators in [DJ].

Let $\mathscr{H}_{0}, \mathscr{H}_{1}$ be infinite dimensional complex Hilbert spaces. Let $H_{0}$ and $A_{0}$ be self-adjoint operators in $\mathscr{H}_{0}$ such that $H_{0}$ is bounded, $H_{0} \in$ $\mathcal{C}^{2}\left(A_{0}\right)$, and such that the strict Mourre estimate (1.1) with $K=0$ holds true for $H_{0}$ and $A_{0}$ on some bounded, infinite interval $\mathcal{I}$. For instance, we can take suitably a bounded, infinite interval $\mathcal{I}$ included in $] 0 ;+\infty[$, $\mathscr{H}_{0}=\mathrm{L}^{2}\left(\mathbb{R}^{d}\right), H_{0}$ a smooth, increasing, and bounded function of the Laplacian on $\mathbb{R}^{d}$, and $A_{0}$ the generator of dilation in $\mathbb{R}^{d}$ (cf. $\mathrm{ABG}, \mathrm{M}$ ). Let $A_{1}$ be self-adjoint operator in $\mathscr{H}_{1}$. Let $\left(g_{n}\right)_{n}$ be a bounded sequence in $\mathcal{D}\left(A_{1}^{2}\right)$ of independent vectors such that it is bounded for the graph norm of $A_{1}^{2}$. Let $\left(\alpha_{n}\right)_{n} \in \ell^{1}$, a sequence of nonzero reals. The serie $\left(\sum_{n \geq 0} \alpha_{n}\left|g_{n}\right\rangle\left\langle g_{n}\right|\right)_{n}$ converge absolutely in the Banach space of bounded operators on $\mathscr{H}_{1}$. Let $C$ be its sum. It is a self-adjoint, compact operator of infinite rank. By Proposition 2.1 each $\alpha_{n}\left|g_{n}\right\rangle\left\langle g_{n}\right| \in \mathcal{C}^{2}\left(A_{1}\right)$ and $\left(\sum_{n>0} \alpha_{n}\left[\left|g_{n}\right\rangle\left\langle g_{n}\right|, A_{1}\right]\right)_{n}$ converges absolutely, since $\left(\left\|g_{n}\right\|\right)_{n}$ and $\left(\left\|A_{1} g_{n}\right\|\right)_{n}$ are bounded. By Proposition 2.2. $C \in \mathcal{C}^{1}\left(A_{1}\right)$ and $\left[C, A_{1}\right]=$ $\sum_{n=0}^{\infty} \alpha_{n}\left[\left|g_{n}\right\rangle\left\langle g_{n}\right|, A_{1}\right]$. Applying this argument again, this implies that $C \in \mathcal{C}^{2}\left(A_{1}\right)$. Let $\lambda \in \stackrel{\circ}{\mathcal{I}}$. We can choose $\left(\alpha_{n}\right)_{n}$ such that $[\lambda-\|C\| ; \lambda+$ $\|C\|] \subset \mathcal{I}$. Let $H_{1}=\lambda+C$. Let $H$ be the bounded self-adjoint operator acting in $\mathscr{H}:=\mathscr{H}_{0} \oplus \mathscr{H}_{1}$ by $H_{0} \oplus H_{1}$. Let $A$ be the self-adjoint operator acting in $\mathscr{H}$ by $A_{0} \oplus A_{1}$. Since $[H, i A]=\left[H_{0}, i A_{0}\right] \oplus\left[C ; i A_{1}\right]$ as form on $\mathcal{D}(A) \times \mathcal{D}(A)$, the regularity of $H_{0}$ w.r.t. $A_{0}$ and the regularity of $C$ w.r.t. $A_{1}$ imply that $H \in \mathcal{C}^{2}(A)$. Since $\operatorname{Ran} C$ is infinite dimensional and the spectrum of $H_{1}$ is contained in $\mathcal{I}$, the point spectrum of $H$ in $\mathcal{I}$ is infinite therefore the Mourre estimate (1.1) cannot hold true 
on $\mathcal{I}$ by Remark 1.1. Since the strict Mourre estimate for $H_{0}$ holds true on $\mathcal{I}, H_{0}$ has no eigenvalue in $\mathcal{I}$ by Remark 1.1. Let $P$ be the orthogonal projection onto the pure point spectral subspace of $H$. By the previous properties, $P^{\perp}[H, i A] P^{\perp}=P^{\perp}\left(\left[H_{0}, i A_{0}\right] \oplus 0\right) P^{\perp}$. Thus the strict Mourre estimate for $H_{0}$ on $\mathcal{I}$ implies the strict, projected Mourre estimate (4.28) for $H$ on $\mathcal{I}$.

\section{Appendix A. Symbolic calculus.}

In this section, we recall well known facts on symbolic calculus and almost analytic extensions (see [DG][Appendix C]). We also show that some sequences of functions used in the main text are bounded in some symbol class.

For $\rho \in \mathbb{R}$, let $\mathcal{S}^{\rho}$ be the class of function $\varphi \in \mathcal{C}^{\infty}(\mathbb{R} ; \mathbb{C})$ such that

$$
\forall k \in \mathbb{N}, \quad C_{k}(\varphi):=\sup _{t \in \mathbb{R}}\langle t\rangle^{-\rho+k}\left|\partial_{t}^{k} \varphi(t)\right|<\infty .
$$

We also write $\varphi^{(k)}$ for $\partial_{t}^{k} \varphi$. Equiped with the semi-norms defined by (A.1), $\mathcal{S}^{\rho}$ is a Fréchet space. Leibniz' formula implies the continuous embedding:

$$
\mathcal{S}^{\rho} \cdot \mathcal{S}^{\rho^{\prime}} \subset \mathcal{S}^{\rho+\rho^{\prime}}
$$

For the functional calculus of the operator $A$ (see (B.1)), we shall use the following result in $[\mathrm{DG}]$ on almost analytic extension.

Lemma A.1. Let $\varphi \in \mathcal{S}^{\rho}$ with $\rho \in \mathbb{R}$. For all $l \in \mathbb{N}$, there is a smooth function $\varphi^{\mathbb{C}}: \mathbb{C} \rightarrow \mathbb{C}$, call an almost analytic extension of $\varphi$, such that:

$$
\begin{aligned}
\left.\varphi^{\mathbb{C}}\right|_{\mathbb{R}}=\varphi, \quad & \left|\frac{\partial \varphi^{\mathbb{C}}}{\partial \bar{z}}(z)\right| \leq c_{1}\langle\operatorname{Re}(z)\rangle^{\rho-1-l}|\operatorname{Im}(z)|^{l} \\
& \operatorname{supp} \varphi^{\mathbb{C}} \subset\left\{x+i y|| y \mid \leq c_{2}\langle x\rangle\right\}, \\
& \varphi^{\mathbb{C}}(x+i y)=0, \text { if } x \notin \operatorname{supp} \varphi .
\end{aligned}
$$

for constants $c_{1}, c_{2}$ depending on the semi-norms (A.1) of $\varphi$ in $\mathcal{S}^{\rho}$.

The function $\chi_{R}$, given by (3.9), belongs to $\mathcal{S}^{\rho}$, for any $\rho$ and any $R$. But we need to know that the family $\left(\chi_{R}\right)_{R \geq 1}$ is bounded in some $\mathcal{S}^{\rho}$.

Lemma A.2. Let $\tau \in \mathcal{C}^{\infty}(\mathbb{R} ; \mathbb{R})$ such that $\tau^{\prime} \in \mathcal{C}_{c}^{\infty}\left(\mathbb{R}^{*} ; \mathbb{R}\right)$. Then the family $\left(\tau_{R}\right)_{R>1}$, with $\tau_{R}(x):=\tau(x / R)$, is bounded in $\mathcal{S}^{0}$. 
Proof. Let $k \in \mathbb{N}$. The semi-norm $C_{k}(\tau)$ (cf. (A.1)) is bounded above by the $\left(\sup \operatorname{supp} \tau^{(k)}\right)^{k}$ times the $L^{\infty}$-norm of $\tau^{(k)}$. For $R>1$ and $t \in \mathbb{R}$,

$$
|t|^{k} \cdot\left|\left(\tau_{R}\right)^{(k)}(t)\right|=(|t| / R)^{k} \cdot\left|\tau^{(k)}(t / R)\right| \leq C_{k}(\tau) .
$$

Thus $\left(\tau_{R}\right)_{R>1}$ is bounded in $\mathcal{S}^{0}$.

Concerning the functions defined in (3.9), (3.14) and just after (3.14), we have the

Corollary A.3. Lemma A.2 applies to $\tau=\chi, \tilde{\chi}, \tilde{\chi}_{\sigma},\left(\tilde{\chi}_{\sigma}\right)^{1 / 2},\left(\sigma \tilde{\chi}_{\sigma}^{\prime}\right)^{1 / 2}$, for $\sigma \in\{-;+\}$, and also to their derivatives.

We now focus on the functions $\psi_{\sigma, R}$, defined in (3.19).

Lemma A.4. The family $\left(\psi_{\sigma, R}^{1 / 2}\right)_{R>1}$ is bounded in $\mathcal{S}^{0}$.

Proof. By (3.19),

$$
\psi_{\sigma, R}^{1 / 2}(x)=\left(\sigma \chi_{\sigma}^{\prime}\right)^{1 / 2}(x / R)\left(h-\sigma x\langle x\rangle^{-2 s}\right)^{1 / 2} \chi_{\sigma}^{1 / 2}(x / R),
$$

for all $x \in \mathbb{R}$ and all $R>1$. By definition of $h$ (cf. (3.15) ), $x \mapsto$ $\left(h-\sigma x\langle x\rangle^{-2 s}\right)^{1 / 2}$ belongs to $\mathcal{S}^{0}$. Now the result follows from Corollary A.3 and (A.2).

\section{Appendix B. Commutator expansions.}

In this section, we recall Helffer-Sjöstrand's functional calculus (cf. [HeS, DG]) and commutator expansions (cf. [DG]).

Let $\rho<0$ and $\varphi \in \mathcal{S}^{\rho}$. The bounded operator $\varphi(A)$ can be recover by Helffer-Sjöstrand's formula:

$$
\varphi(A)=\frac{i}{2 \pi} \int_{\mathbb{C}} \frac{\partial \varphi^{\mathbb{C}}}{\partial \bar{z}}(z-A)^{-1} d z \wedge d \bar{z}
$$

where the integral exists in the norm topology, by A.3 with $l=1$. This can be extended as shown in

Lemma B.1. Let $k \in \mathbb{N}, \rho<k$ and $\varphi \in \mathcal{S}^{\rho}$. Strongly in $\mathcal{D}\left(\langle A\rangle^{k}\right)$, (B.1) holds true.

Proof. Let $f \in \mathcal{D}\left(\langle A\rangle^{k}\right) \subset \mathcal{D}(\varphi(A))$ and $\chi_{R}$ be like in (3.9), then

$$
\varphi(A) \chi_{R}(A) f=\frac{i}{2 \pi} \int_{\mathbb{C}} \frac{\partial\left(\varphi_{k} \chi_{R}\right)^{\mathbb{C}}}{\partial \bar{z}}(z-A)^{-1}\langle A\rangle^{k} f d z \wedge d \bar{z}
$$


where $\varphi_{k}(t):=\varphi(t)\langle t\rangle^{-k}$ belongs to $\mathcal{S}^{\rho-k}$. By Lemma A.2 and (A.2), $\left(\varphi_{k} \chi_{R}\right)_{R}$ is bounded in $\mathcal{S}^{\rho-k}$. Since $\rho-k<0$, the result follows from (A.3) with $l=1$ and the dominated convergence theorem.

Notice that, for some $c>0$ and $s \in[0 ; 1[$, there exists some $C>0$ such that, for all $z=x+i y \in\{a+i b|0<| b \mid \leq c\langle a\rangle\}$ (like in (A.4)),

$$
\left\|\langle A\rangle^{s}(A-z)^{-1}\right\| \leq C\langle x\rangle^{s} \cdot|y|^{-1} \text {. }
$$

Next we come to a commutator expansion.

Proposition B.2. Let $k \in \mathbb{N}^{*}$ and $B$ be a self-adjoint and bounded operator in $\mathcal{C}^{k}(A)$. Let $\rho<k$ and $\varphi \in \mathcal{S}^{\rho}$. In the sense of forms on $\mathcal{D}\left(\langle A\rangle^{k-1}\right) \times \mathcal{D}\left(\langle A\rangle^{k-1}\right)$ :

$$
\begin{array}{r}
{[\varphi(A), B]=\sum_{j=1}^{k-1} \frac{1}{j !} \varphi^{(j)}(A) \operatorname{ad}_{A}^{j}(B)} \\
+\frac{i}{2 \pi} \int_{\mathbb{C}} \frac{\partial \varphi^{\mathbb{C}}}{\partial \bar{z}}(z-A)^{-k} \operatorname{ad}_{A}^{k}(B)(z-A)^{-1} d z \wedge d \bar{z} .
\end{array}
$$

In particular, if $\rho<1$, then $B \in \mathcal{C}^{1}(\varphi(A))$.

Proof. Thanks to Lemma B.1, we can write, as form on $\mathcal{D}\left(\langle A\rangle^{k}\right) \times$ $\mathcal{D}\left(\langle A\rangle^{k}\right)$ :

$$
\begin{aligned}
{[\varphi(A), B] } & =\frac{i}{2 \pi} \int_{\mathbb{C}} \frac{\partial \varphi^{\mathbb{C}}}{\partial \bar{z}}(z-A)^{-1} \operatorname{ad}_{A}(B)(z-A)^{-1} d z \wedge d \bar{z} \\
& =\sum_{j=1}^{k-1} \frac{i}{2 \pi} \int_{\mathbb{C}} \frac{\partial \varphi^{\mathbb{C}}}{\partial \bar{z}}(z-A)^{-j-1} \operatorname{ad}_{A}^{j}(B) d z \wedge d \bar{z} \\
& +\frac{i}{2 \pi} \int_{\mathbb{C}} \frac{\partial \varphi^{\mathbb{C}}}{\partial \bar{z}}(z-A)^{-k} \operatorname{ad}_{A}^{k}(B)(z-A)^{-1} d z \wedge d \bar{z} .
\end{aligned}
$$

This yields (B.4) on $\mathcal{D}\left(\langle A\rangle^{k}\right) \times \mathcal{D}\left(\langle A\rangle^{k}\right)$. Since $B \in \mathcal{C}^{k}(A)$, the commutators $\operatorname{ad}_{A}^{j}(B)$, for $1 \leq j \leq k$, are bounded. Now, as in the proof of Lemma B.1, we see that this form extends to a bounded form on $\mathcal{D}\left(\langle A\rangle^{k-1}\right) \times \mathcal{D}\left(\langle A\rangle^{k-1}\right)$ since the $\varphi^{(j)}$ belong to $\mathcal{S}^{\rho-1}$.

The rest of the previous expansion is estimated in

Lemma B.3. Let $B \in \mathcal{C}^{k}(A)$ self-adjoint and bounded. Let $\varphi \in \mathcal{S}^{\rho}$, with $\rho<k$. Let $I_{k}(\varphi)$ the rest of the development of order $k$ (B.4) of $[\varphi(A), B]$, namely (B.5). Let $s, s^{\prime} \geq 0$ such that $s^{\prime}<1, s<k$, and $\rho+s+s^{\prime}<k$. Then $\langle A\rangle^{s} I_{k}(\varphi)\langle A\rangle^{s^{\prime}}$ is bounded and it is uniformly bounded when $\varphi$ stays in a bounded subset of $\mathcal{S}^{\rho}$. In particular, $I_{k}(\varphi)$ 
is a bounded operator. Let $R>0$. If $\varphi$ stays in a bounded subset of $\left\{\psi \in \mathcal{S}^{\rho} \mid[-R ; R] \cap \operatorname{supp}(\varphi)=\emptyset\right\}$ then $\langle R\rangle^{k-\rho-s-s^{\prime}}\left\|\langle A\rangle^{s} I_{k}(\varphi)\langle A\rangle^{s^{\prime}}\right\|$ is uniformly bounded.

Proof. We will follow ideas from DG] [Lemma C.3.1]. In this proof, all the constants are denoted by $C$, independently of their value. Given a complex number $z, x$ and $y$ will denote its real and imaginary part, respectively. Since $B \in \mathcal{C}^{k}(A), \operatorname{ad}_{A}^{k}(B)$ is bounded. We start with the second assertion. Let $\varphi \in \mathcal{S}^{\rho}, R>0$ such that $[-R ; R] \cap \operatorname{supp}(\varphi)=\emptyset$. Notice that, by (A.5), $\varphi^{\mathbb{C}}(x+i y)=0$ for $|x| \leq R$. By definition of $I_{k}$, we consider (B.5) and switch to the variable $(x, y)$ by noticing that $d z \wedge d \bar{z}=-2 i d x \wedge d y$. By (B.3),

$$
\begin{array}{r}
\left\|\langle A\rangle^{s} I_{k}(\varphi)\langle A\rangle^{s^{\prime}}\right\| \leq \frac{1}{\pi} \int\left|\frac{\partial \varphi^{\mathbb{C}}}{\partial \bar{z}}\right| \cdot \frac{\langle x\rangle^{s}}{|y|^{k}} \cdot\left\|\operatorname{ad}_{A}^{k}(B)\right\| \cdot \frac{\langle x\rangle^{s^{\prime}}}{|y|} d x \wedge d y \\
\leq C(\varphi) \int_{|x| \geq R} \int_{|y| \leq c_{2}\langle x\rangle}\langle x\rangle^{\rho+s+s^{\prime}-1-l}|y|^{l}|y|^{-k-1} d x \wedge d y
\end{array}
$$

for any $l$, by (A.3. . We choose $l=k+1$. We have,

$$
\begin{aligned}
\left\|\langle A\rangle^{s} I_{k}(\varphi)\langle A\rangle^{s^{\prime}}\right\| & \leq C(\varphi) \int_{|x| \geq R}\langle x\rangle^{\rho+s+s^{\prime}-k-1} d x \\
& \leq C(\varphi)\langle R\rangle^{\rho+s+s^{\prime}-k} .
\end{aligned}
$$

Since $C(\varphi)$ is bounded when $\varphi$ stays in a bounded subset of $\mathcal{S}^{\rho}$, this yields the second assertion. For the first one, we can follow the same lines, replacing $R$ by 0 in the integrals, and arrive at the result.

\section{Appendix C. Technical estimates.}

Lemma C.1. Let $\varepsilon \in] 0,1-s\left[\right.$ and suppose $B \in \mathcal{C}^{2}(A)$ bounded and self-adjoint. Then, for all $f \in \mathscr{H}$,

$$
\begin{gathered}
\left\langle\tilde{\chi}_{R}(A) f,\left[B,-A\langle A\rangle^{-2 s}\right] \tilde{\chi}_{R}(A) f\right\rangle \geq O_{R}\left(R^{-\varepsilon}\right)\left\|\tilde{\chi}_{R}(A)\langle A\rangle^{-s} f\right\|^{2} \\
+(2 s-1)\left\langle\tilde{\chi}_{R}(A)\langle A\rangle^{-s} f,[B, A] \tilde{\chi}_{R}(A)\langle A\rangle^{-s} f\right\rangle .
\end{gathered}
$$

Proof. Let $D=\left[B,-\langle A\rangle^{-2 s} A\right]-\left(2 s A^{2}\langle A\rangle^{-2 s-2}-\langle A\rangle^{-2 s}\right)[B, i A]$. By Lemma B.3 for $k=2$, as $t \mapsto\langle t\rangle^{-2 s} t \in \mathcal{S}^{1-2 s}$, one has $\langle A\rangle^{s+\varepsilon} D\langle A\rangle^{s}$ bounded for $\varepsilon<1$. Then, using the fact that $\tilde{\chi}_{R / 2}(t)=1$ for $t$ in the support of $\tilde{\chi}_{R}$,

$$
\begin{aligned}
\left\langle\tilde{\chi}_{R}(A) f, D \tilde{\chi}_{R}(A) f\right\rangle & =\left\langle\langle A\rangle^{-\varepsilon} \tilde{\chi}_{R / 2}(A) \tilde{\chi}_{R}(A)\langle A\rangle^{-s} f,\langle A\rangle^{s+\varepsilon} D \tilde{\chi}_{R}(A) f\right\rangle \\
& \leq O\left(R^{-\varepsilon}\right) \cdot\left\|\tilde{\chi}_{R}(A)\langle A\rangle^{-s} f\right\|^{2} .
\end{aligned}
$$


Since $[B, A] \in \mathcal{C}^{1}(A)$ and $t \mapsto\langle t\rangle^{-s} \in \mathcal{S}^{-s}$, Lemma B.3 gives that $\langle A\rangle^{s+\varepsilon}\left[\langle A\rangle^{-s},[B, A]\right]\langle A\rangle^{s}$ bounded for $\varepsilon<1-s$. Using, like above, the contribution of $\tilde{\chi}_{R / 2}(A)$,

$$
\begin{aligned}
\mid\left\langle\tilde{\chi}_{R}(A) f,\right. & \left.\left(2 s A^{2}\langle A\rangle^{-2 s-2}-\langle A\rangle^{-2 s}\right)[B, i A] \tilde{\chi}_{R}(A) f\right\rangle \\
& -\left\langle\tilde{\chi}_{R}(A) f,\left(2 s A^{2}\langle A\rangle^{-2}-1\right)\langle A\rangle^{-s}[B, i A]\langle A\rangle^{-s} \tilde{\chi}_{R}(A) f\right\rangle \mid \\
\leq & O\left(R^{-\varepsilon}\right)\left\|\langle A\rangle^{-s} \chi_{R}(A) f\right\|^{2} .
\end{aligned}
$$

To conclude, observe that $\left\|\tilde{\chi}_{R / 2}(A)\left(\mathrm{Id}-A^{2}\langle A\rangle^{-2}\right)\right\|=O\left(R^{-2}\right)$.

Lemma C.2. Let $B \in \mathcal{C}^{2}(A)$ bounded and self-adjoint. For all $f \in \mathscr{H}$,

$$
\begin{aligned}
\mid\left\langle f,\left(\sigma h-A\langle A\rangle^{-2 s}\right)\right. & \left.\tilde{\chi}_{\sigma, R}(A)\left[B, \tilde{\chi}_{\sigma, R}(A)\right] f\right\rangle- \\
& \left\langle f, \tilde{\chi}_{\sigma, R}^{\prime}(A)\left(\sigma h-A\langle A\rangle^{-2 s}\right) \tilde{\chi}_{\sigma, R}(A)[B, A] f\right\rangle \mid \\
\leq & O\left(R^{2 s-2}\right)\left\|\tilde{\chi}_{R}(A)\langle A\rangle^{-s} f\right\| \cdot\left\|\langle A\rangle^{-s} f\right\| .
\end{aligned}
$$

Proof. By Lemma B.3, we develop the commutator and denote the rest by $I_{2}$. Its contribution is

$$
\left\langle\tilde{\chi}_{R / 2}(A)\langle A\rangle^{-s} f,\left(\sigma h-A\langle A\rangle^{-2 s}\right) \tilde{\chi}_{\sigma, R}(A)\langle A\rangle^{s} I_{2}\langle A\rangle^{s}\langle A\rangle^{-s} f\right\rangle .
$$

Note that $\tilde{\chi}_{R / 2}(A) f$ appears freely thanks to the presence of $\tilde{\chi}_{\sigma, R}(A)$. By Corollary A.3. $\left(\tilde{\chi}_{\sigma, R}\right)_{R}$ is bounded in $\mathcal{S}^{0}$. Note also that $[-R, R]$ is not contained in the support of $\tilde{\chi}_{\sigma, R}$. Then, from Lemma B.3, used with $k=2$, we obtain that $\langle A\rangle^{s} I_{2}\langle A\rangle^{s}=O\left(R^{2 s-2}\right)$.

Lemma C.3. For $B \in \mathcal{C}^{1}(A)$ bounded and self-adjoint,

(1) $\left\|C_{R}\langle A\rangle^{\alpha}\right\|=O\left(R^{\alpha}\right)$, for $\alpha \in \mathbb{R}$,

(2) $\left\|\left[B, C_{R}\right]\langle A\rangle^{\alpha}\right\|=O\left(R^{\alpha-1}\right)$, for $0 \leq \alpha<1$.

Proof. Since $\psi_{R}(t)=0$ for $|t| \notin[R, 2 R]$, the point (1) follows. Since $\left(\psi_{R}\right)_{R}$ is bounded in $\mathcal{S}^{0}$ (cf. Lemma A.4) and since $[-R, R]$ is not contained in the support of $\psi_{R}$, we get the point (2) by Lemma B.3.

Lemma C.4. Let $B \in \mathcal{C}^{2}(A)$ bounded, self-adjoint. For all $f \in \mathscr{H}$,

$$
\begin{aligned}
\mid\left\langle C_{R} f,[B, i A] C_{R} f\right\rangle- & \left\langle f, C_{R}^{2}[B, i A] f\right\rangle \mid \\
& \leq O_{R}\left(R^{2 s-1}\right) \cdot\left\|\tilde{\chi}_{R / 2}(A)\langle A\rangle^{-s} f\right\| \cdot\left\|\langle A\rangle^{-s} f\right\| .
\end{aligned}
$$

Proof. Given $f \in \mathscr{H}$ and using (3.20),

$$
\begin{gathered}
\left\langle C_{R} f,[B, i A] C_{R} f\right\rangle=\left\langle f, C_{R}^{2}[B, i A] f\right\rangle \\
-\quad\left\langle\tilde{\chi}_{R / 2}(A)\langle A\rangle^{-s} f, C_{R}\langle A\rangle^{s}\left[C_{R},[B, i A]\right]\langle A\rangle^{s} \cdot\langle A\rangle^{-s} f\right\rangle .
\end{gathered}
$$


The last term is estimated above by

$$
\left\|C_{R}\langle A\rangle^{s}\right\| \cdot\left\|\left[C_{R},[B, A]\right]\langle A\rangle^{s}\right\| \cdot\left\|\tilde{\chi}_{R / 2}(A)\langle A\rangle^{-s} f\right\| \cdot\left\|\langle A\rangle^{-s} f\right\| .
$$

Now Lemma C.3 gives the result.

\section{REFERENCES}

[ABG] W.O. Amrein, A. Boutet de Monvel and V. Georgescu: $C_{0}$-groups, commutator methods and spectral theory of $N$-body hamiltonians., Birkhäuser 1996.

[BFS] V. Bach, J. Fröhlich and I.M. Sigal: Quantum electrodynamics of confined non-relativistic particles. Adv. in Math. 137, 299-395, 1998.

[BCHM] J-F. Bony, R. Carles, D. Haeffner, L. Michel: Scattering theory for the Schrödinger equation with repulsive potential. J. Math. Pures Appl. 84, no. 5, 509-579, 2005.

[B] N. Burq: Semiclassical estimates for the resolvent in non trapping geometries. Int. Math. Res. Notices 2002, no 5, 221-241.

[CJ] F. Castella, Th. Jecko: Besov estimates in the high-frequency Helmholtz equation, for a non-trapping and $C^{2}$ potential. To appear in J. Diff. Eq.

[C] L. Cattaneo: Mourre's inequality and Embedded bounded states, Bull. Sci Math. 129, Issue 7, 591-614, 2005.

[CGH] L. Cattaneo, G. M. Graf and W. Hunziker: A general resonance theory based on Mourre's inequality, math-ph/0507063

[DG] J. Dereziński and C. Gérard: Scattering theory of classical and quantum $N$-particle systems. Springer-Verlag 1997.

[DJ] J. Dereziński and V. Jakšić: Spectral theory of Pauli-Fierz operators. J. Funct. Anal. 180, no 2, pp. 243-327, 2001.

[GGé] V. Georgescu and C. Gérard: On the Virial Theorem in Quantum Mechanics, Commun. Math. Phys. 208, 275-281, (1999).

[GGM1] V. Georgescu, C. Gérard, and J.S. Møller: Commutators, $C_{0}$-semigroups and resolvent estimates, J. Funct. Anal. 216, no 2, pp. 303-361, 2004.

[GGM2] V. Georgescu, C. Gérard, and J.S. Møller: Spectral theory of massless Pauli-Fierz models, Comm. Math. Phys. 249, no 1, pp. 29-78, 2004.

[GGo] V. Georgescu, S. Golénia: Isometries, Fock spaces and spectral analysis of Schrdinger operators on trees., Journal of Functional Analysis 227 (2005), 389-429.

[HeS] B. Helffer and J. Sjöstrand: Opérateurs de Schrödinger avec champs magnétiques faibles et constants. Exposé No. XII, Séminaire EDP, février 1989, Ecole Polytechnique.

[H] L. Hörmander: The analysis of linear partial differential operators II., Springer-Verlag Berlin Heidelberg 1983.

[HuS] W. Hunziker and I.M. Sigal: The quantum $N$-body problem, J. Math. Phys. 41 (6), 3448-3510, 2000.

[J1] Th. Jecko: From classical to semiclassical non-trapping behaviour, C. R. Acad. Sci. Paris, Ser. I, 338, p. 545-548, 2004.

[J2] Th. Jecko : Non-trapping condition for semiclassical Schrödinger operators with matrix-valued potentials. Math. Phys. Electronic Journal, No. 2, vol. 11, 2005. 
[JMP] A. Jensen, E. Mourre, P. Perry: Multiple commutator estimates and resolvent smoothness in quantum scattering theory. Ann. Inst. Henri Poincaré, vol. 41, no 2, 1984, p. 207-225.

[M] E. Mourre: Absence of singular continuous spectrum for certain selfadjoint operators. Commun. in Math. Phys. 78, 391-408, 1981.

[RS4] M. Reed, B. Simon : Methods of Modern Mathematical Physics, Tome IV: Analysis of operators. Academic Press.

[S] J. Sahbani:The conjugate operator method for locally regular Hamiltonians. J. Oper. Theory 38, No. 2, 297-322 (1997).

Departement of Mathematical Methods in Physics, Warsaw UniverSity, Hoża 74, 00-682 Warszawa, Poland

E-mail address: Sylvain.Golenia@fuw.edu.pl

IRMAR, UMR 6625 DU CNRS, Université de Rennes 1, Campus De Beaulieu, 35042, Rennes Cedex France

E-mail address: Thierry.Jecko@univ-rennes1.fr 